Algebraic 83 Geometric $\mathcal{T}$ opology

Volume 1 (2001) 243-270

Published: 23 April 2001

ATG

\title{
Homology cylinders: an enlargement of the mapping class group
}

\author{
JEROME LEVINE
}

\begin{abstract}
We consider a homological enlargement of the mapping class group, defined by homology cylinders over a closed oriented surface (up to homology cobordism). These are important model objects in the recent Goussarov-Habiro theory of finite-type invariants of 3-manifolds. We study the structure of this group from several directions: the relative weight filtration of Dennis Johnson, the finite-type filtration of Goussarov-Habiro, and the relation to string link concordance.

We also consider a new Lagrangian filtration of both the mapping class group and the group of homology cylinders.
\end{abstract}

AMS Classification 57N10; 57M25

Keywords Homology cylinder, mapping class group, clasper, finite-type invariant

\section{Introduction}

The mapping class group $\Gamma_{g}$ is the group of diffeotopy classes of orientation preserving diffeomorphisms of the closed oriented surface $\Sigma_{g}$ of genus $g$. There has been a great deal of work aimed at the determination of the algebraic structure of this group. For example, some years ago D. Johnson defined a filtration on $\Gamma_{g}$ (the relative weight filtration) and observed that the associated graded group is a Lie subalgebra of a Lie algebra $\mathrm{D}(H)$ constructed explicitly from $H=H_{1}\left(\Sigma_{g}\right)$. Johnson, Morita and others (see $[\mathrm{J}],[\mathrm{Mo}],[\mathrm{M}]$ ) have investigated this Lie subalgebra but its precise determination is still open. R. Hain [Hn] has studied the lower central series filtration of the Torelli group (the subgroup of $\Gamma_{g}$ whose elements are homologically trivial) and found a simple explicit presentation over $\mathbb{Q}$. We also mention the work of Oda (see $[\mathrm{O}],[\mathrm{L}])$ relating the pure braid group to $\Gamma_{g}$.

One reason for interest in $\Gamma_{g}$ is that it is related in an obvious way to the structure of 3-manifolds via the Heegard construction. From this viewpoint, the 
subgroup $\Gamma_{g}^{B}$ consisting of diffeomorphisms which extend over the handlebody $T_{g}$ of genus $g$, and the coset space $\Gamma_{g} / \Gamma_{g}^{B}$, are of obvious interest.

In this note we propose a "homological" generalization of these groups, where we replace $\Gamma_{g}$ by a group $\mathcal{H}_{g}$ of homology bordism classes of homology cylinders over $\Sigma_{g}$. Homology cylinders have appeared and been studied in recent work of Goussarov [Go] and Habiro [H], as important model objects for their new theory of finite-type invariants of general 3 -manifolds. We will see that $\Gamma_{g}$ is a subgroup of $\mathcal{H}_{g}$. Furthermore the notion of Heegard construction translates to the more general context of homology cylinders - the relevant subgroup is now $\mathcal{H}_{g}^{B}$, consisting of those homology cylinders which extend to homology cylinders over $T_{g}$.

It turns out that the structure of $\mathcal{H}_{g}$ presents different problems than $\Gamma_{g}$. The relative weight filtration extends to a filtration of $\mathcal{H}_{g}$ but now the associated graded group is all of $\mathrm{D}(H)$. On the other hand the residue of the filtration (i.e. the intersection of the subgroups of the filtration) is non-trivial (it is trivial for $\Gamma_{g}$ ). There are some approaches to the study of $\mathcal{H}_{g}$ which don't seem to have useful analogs for $\Gamma_{g}$. Using the recent work of Goussarov [Go] and Habiro $[\mathrm{H}]$, there is a notion of finite-type invariants for homology cylinders. We will show, using results announced by Habiro $[\mathrm{H}]$, that this is entirely captured by the relative weight filtration except for one piece in degree 1 , which is a $\mathbb{Z} / 2$-vector space defined by a natural generalization of the Birman-Craggs homomorphisms. In a different direction we will exhibit a close relationship between $\mathcal{H}_{g}$ and a framed string link concordance group $\mathcal{S}_{g}^{\mathrm{fr}}$ - extending the natural map from the pure braid group into $\Gamma_{g}$ defined by Oda. We will see that $\mathcal{S}_{g}^{\text {fr }}$ maps into $\mathcal{H}_{g}$, inducing a bijection of $\mathcal{S}_{g}^{\text {fr }}$ with the coset space $\overline{\mathcal{H}}_{g} / \mathcal{H}_{g}^{B}$, where $\overline{\mathcal{H}}_{g}$ is a natural subset of $\mathcal{H}_{g}$ containing the "Torelli" subgroup -it is interesting to note that there is no analogous result for $\Gamma_{g}$.

The inclusion $\Gamma_{g}^{B} \subseteq \Gamma_{g}$ can be illuminated by the introduction of a new filtration of $\Gamma_{g}$ which we call the Lagrangian filtration. The residue of this filtration is exactly $\Gamma_{g}^{B}$ and the associated graded group imbeds in a Lie algebra $\mathrm{D}(L)$ constructed from the Lagrangian subgroup $L=\operatorname{Ker}\left\{H_{1}\left(\Sigma_{g}\right) \rightarrow H_{1}\left(T_{g}\right)\right\}$. Determination of the image seems as difficult as the analogous problem for the relative weight filtration. As in the case of the relative weight filtration, the associated graded group for the Lagrangian filtration of $\mathcal{H}_{g}$ is isomorphic to $\mathrm{D}(L)$. However the residue of the filtration turns out to be larger than $\mathcal{H}_{g}^{B}$.

I would like to thank Stavros Garoufalidis for many useful discussions.

This work was partially supported by NSF grant DMS-99-71802 and by an Israel-US BSF grant. 


\section{Homology cylinders}

\subsection{Preliminaries}

For the usual technical reasons it will be easier to work with the punctured surface. Let $\Sigma_{g, 1}$ denote the compact orientable surface of genus $g$ with one boundary component. A homology cylinder over $\Sigma_{g, 1}$ is a compact orientable 3 -manifold $M$ equipped with two imbeddings $i^{-}, i^{+}: \Sigma_{g, 1} \rightarrow \partial M$ so that $i^{+}$is orientation-preserving and $i^{-}$is orientation-reversing and if we denote $\Sigma^{ \pm}=$ $\operatorname{Im} i^{ \pm}\left(\Sigma_{g, 1}\right)$, then $\partial M=\Sigma^{+} \cup \Sigma^{-}$and $\Sigma^{+} \cap \Sigma^{-}=\partial \Sigma^{+}=\partial \Sigma^{-}$. We also require that $i^{ \pm}$be homology isomorphisms. This notion is introduced in $[\mathrm{H}]$, using the terminology homology cobordism. We can multiply two homology cylinders by identifying $\Sigma^{-}$in the first with $\Sigma^{+}$in the second via the appropriate $i^{ \pm}$. Thus $\mathcal{C}_{g}$, the set of orientation-preserving diffeomorphism classes of homology cylinders over $\Sigma_{g, 1}$ is a semi-group whose identity is the product $I \times \Sigma_{g, 1}$, with $\Sigma^{-}=0 \times \Sigma_{g, 1}, \Sigma^{+}=1 \times \Sigma_{g, 1}$, with their collars stretched half-way along $I \times \partial \Sigma_{g, 1} \cdot \mathcal{C}_{g}$ is denoted $\mathcal{C}\left(\Sigma_{g}\right)$ in $[\mathrm{H}]$.

There is a canonical homomorphism $\Gamma_{g, 1} \rightarrow \mathcal{C}_{g}$ that sends $\phi$ to $(M=I \times$ $\left.\Sigma_{g, 1}, i^{-}=0 \times \mathrm{id}, i^{+}=1 \times \phi\right)$. Nielsen [N] showed that the natural map $\eta: \Gamma_{g, 1} \rightarrow$ $\operatorname{Aut}_{0}(F)$ is an isomorphism, where $F$ is the free group on $2 g$ generators $\left\{x_{i}, y_{i}\right\}$, identified with the fundamental group of $\Sigma_{g, 1}$ (with base-point on $\partial \Sigma_{g, 1}$ ) - see Figure 1-and $\operatorname{Aut}_{0}(F)$ is the group of automorphisms of $F$ which fix the element $\omega_{g}=\left(y_{1} \cdots y_{g}\right)^{-1}\left(x_{1} y_{1} x_{1}^{-1} \cdots x_{g} y_{g} x_{g}^{-1}\right)$, representing the boundary of $\Sigma_{g, 1}$.

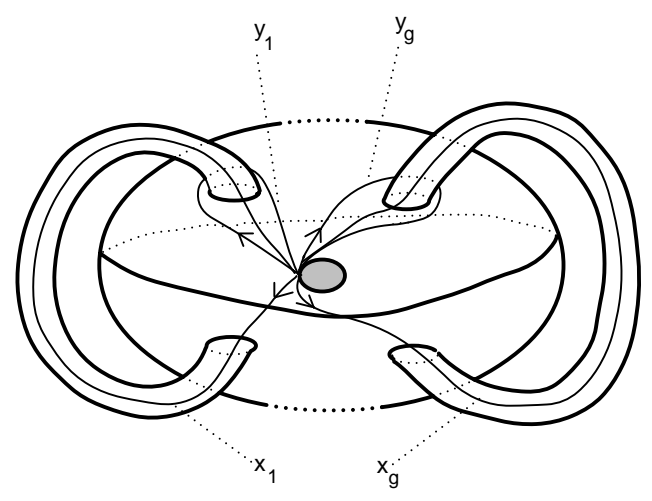

Figure 1: Generators of $\pi_{1}\left(\Sigma_{g, 1}\right)$

We can convert $\mathcal{C}_{g}$ into a group $\mathcal{H}_{g}$ by considering homology bordism classes of homology cylinders. If $M, N$ are homology cylinders, we can construct a 
closed manifold $W=M \cup(-N)$, where $\Sigma^{ \pm}$of $M$ is attached to $\Sigma^{\mp}$ of $N$ via their identifications with $\Sigma_{g, 1}$. A homology bordism between $M$ and $N$ is a manifold $X$ such that $\partial X=W$ and the inclusions $M \subseteq X, N \subseteq X$ are homology equivalences - we say that $M$ and $N$ are homology bordant. This is an equivalence relation, since we can paste two homology bordisms together to create a third. Furthermore the multiplication of homology cylinders preserves homology bordism classes - see Figure 2. For any homology cylinder $M$ we can

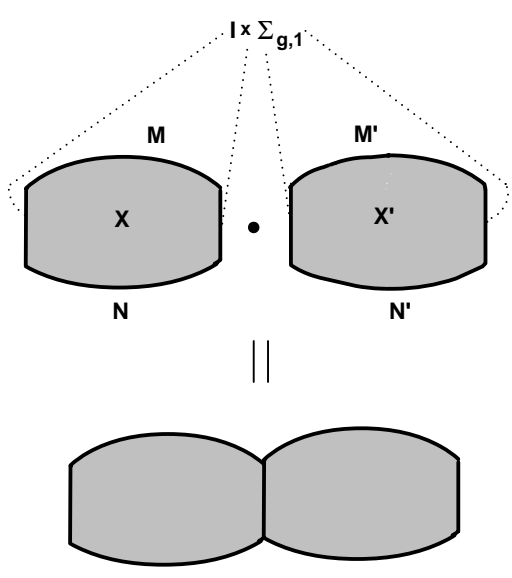

Figure 2: Homology bordism invariance of multiplication of homology cylinders

also consider $-M$ as a homology cylinder with the roles of $\Sigma^{ \pm}$reversed. Then $I \times M$ is a homology bordism between the product $M \cdot(-M)=\partial I \times M \cup I \times \Sigma^{-}$ and $I \times \Sigma_{g, 1}=I \times \Sigma^{+}$, the identity element of $\mathcal{C}_{g}$. Thus $\mathcal{H}_{g}$ is a group.

For any group $G$, let $G_{q}$ denote the subgroup generated by commutators of order $q$. In [GL] the isomorphism $\eta: \Gamma_{g, 1} \cong \operatorname{Aut}_{0}(F)$ is extended to a sequence of maps

$$
\eta_{k}: \mathcal{H}_{g} \rightarrow \operatorname{Aut}_{0}\left(F / F_{k+1}\right)
$$

for $k \geq 1$, which are consistent in the obvious sense. $\operatorname{Aut}_{0}\left(F / F_{q}\right)$ consists of all automorphisms of $F / F_{q}$ which satisfy the equation

$$
\left[h\left(x_{1}\right), h\left(y_{1}\right)\right] \cdots\left[h\left(x_{g}\right), h\left(y_{g}\right)\right]=\left[x_{1}, y_{1}\right] \cdots\left[x_{g}, y_{g}\right] \bmod F_{q+1}
$$

For example $\operatorname{Aut}_{0}\left(F / F_{2}\right)$ is just the symplectic group $\operatorname{Sp}(H)$, where $H=$ $H_{1}\left(\Sigma_{g, 1}\right)$.

For the reader's convenience we recall the definition of $\eta_{k}$. Given $\left(M, i^{+}, i^{-}\right) \in$ $\mathcal{H}_{g}$ consider the homomorphisms $i_{*}^{ \pm}: F \rightarrow \pi_{1}(M)$, where the base-point is taken in $\partial \Sigma^{+}=\partial \Sigma^{-}$. In general, $i_{*}^{ \pm}$are not isomorphisms-however, since 
$i^{ \pm}$are homology isomorphisms, it follows from Stallings [St] that they induce isomorphisms $i_{n}^{ \pm}: F / F_{n} \rightarrow \pi_{1}(M) / \pi_{1}(M)_{n}$ for any $n$. We then define $\eta_{k}\left(M, i^{ \pm}\right)=\left(i_{k+1}^{-}\right)^{-1} \circ i_{k+1}^{+}$. It is easy to see that $\eta_{k}\left(M, i^{ \pm}\right) \in \operatorname{Aut}_{0}\left(F / F_{k+1}\right)$.

One consequence of the existence of these maps is that the map $\Gamma_{g, 1} \rightarrow \mathcal{H}_{g}$ is injective. Furthermore, the following theorem is proved in [GL].

Theorem 1 Every $\eta_{k}$ is onto.

We now define a filtration of $\mathcal{H}_{g}$ by setting $\mathcal{F}_{k}^{w}\left(\mathcal{H}_{g}\right)=\operatorname{Ker} \eta_{k}$. Then $\mathcal{F}_{k}^{w}\left(\mathcal{H}_{g}\right) \cap$ $\Gamma_{g, 1}=\Gamma_{g, 1}[k]$ is the standard relative weight filtration of $\Gamma_{g, 1}$ (this is denoted $\mathcal{M}(k+1)$ in $[\mathrm{Mo}])$. Let $\mathcal{G}_{k}^{w}\left(\mathcal{H}_{g}\right)=\mathcal{F}_{k}^{w}\left(\mathcal{H}_{g}\right) / \mathcal{F}_{k+1}^{w}\left(\mathcal{H}_{g}\right)$. There is, for every $k \geq 1$, a short exact sequence

$$
1 \rightarrow \mathrm{D}_{k}(H) \rightarrow \operatorname{Aut}_{0}\left(F / F_{k+2}\right) \rightarrow \operatorname{Aut}_{0}\left(F / F_{k+1}\right) \rightarrow 1
$$

where $\mathrm{D}_{k}(H)$ is the kernel of the bracketing map $H \otimes \mathrm{L}_{k+1}(H) \rightarrow \mathrm{L}_{k+2}(H)$. $\mathrm{L}_{q}(H)$ is the degree $q$ part of the free Lie algebra over $H$ (see [GL]). Theorem 1 has, as an immediate consequence:

Corollary 2.1 $\eta_{k+1}$ induces an isomorphism $J_{k}^{H}: \mathcal{G}_{k}^{w}\left(\mathcal{H}_{g}\right) \cong \mathrm{D}_{k}(H)$ for $k \geq$ 1.

Note that the induced monomorphism $J_{k}: \Gamma_{g, 1}[k] / \Gamma_{g, 1}[k+1] \rightarrow \mathrm{D}_{k}(H)$ is not generally onto - computing its image is a fundamental problem in the study of the mapping class group (see $[\mathrm{J}],[\mathrm{Mo}],[\mathrm{M}])$. We will see that $\mathcal{F}_{\infty}^{w}\left(\mathcal{H}_{g}\right)=$ $\bigcap_{k} \mathcal{F}_{k}^{w}\left(\mathcal{H}_{g}\right)$ is non-trivial whereas it follows from Nielsen's theorem that $\Gamma_{g, 1}[\infty]=\{1\}$.

\subsection{Filtrations of the Torelli group}

Since $\Gamma_{g, 1}[1]=T_{g}$ is the classical Torelli group, we will refer to $\mathcal{F}_{1}^{w}\left(\mathcal{H}_{g}\right)=\mathcal{T H}_{g}$ as the homology Torelli group.

It is pointed out in $[\mathrm{Mo}]$ that

$$
\left[\Gamma_{g, 1}[k], \Gamma_{g, 1}[l]\right] \subseteq \Gamma_{g, 1}[k+l]
$$

Thus $\left(T_{g}\right)_{k} \subseteq \Gamma_{g, 1}[k]$. Furthermore the associated graded groups $\mathcal{G}_{*}^{l}\left(T_{g}\right)$ and $\mathcal{G}_{*}^{w}\left(\Gamma_{g, 1}\right)$, where $\mathcal{G}_{k}^{l}\left(T_{g}\right)=\left(T_{g}\right)_{k} /\left(T_{g}\right)_{k+1}$ and $\mathcal{G}_{k}^{w}\left(\Gamma_{g, 1}\right)=\Gamma_{g, 1}[k] / \Gamma_{g, 1}[k+1]$, are graded Lie algebras with bracket defined by the commutator and we have a Lie algebra homomorphism $j: \mathcal{G}_{*}^{l}\left(T_{g}\right) \rightarrow \mathcal{G}_{*}^{w}\left(\Gamma_{g, 1}\right)$ induced by the inclusions. Also $D_{*}(H)$ is a Lie algebra, as described in $[\mathrm{M}]$, and the inclusion $J: \mathcal{G}_{*}^{w}\left(\Gamma_{g, 1}\right) \hookrightarrow$ 
$D_{*}(H)$ is a Lie algebra homomorphism. Johnson $[\mathrm{J}]$ shows that, after $\otimes \mathbb{Q}$, both $j$ and $J$ are isomorphisms at the degree 1 level.

$$
\mathcal{G}_{1}^{l}\left(T_{g}\right) \otimes \mathbb{Q} \stackrel{\cong}{\longrightarrow} \mathcal{G}_{1}^{w}\left(\Gamma_{g, 1}\right) \otimes \mathbb{Q} \stackrel{\cong}{\longrightarrow} D_{1}(H) \otimes \mathbb{Q}
$$

Thus $\operatorname{Im} j \otimes \mathbb{Q}$ and $\operatorname{Im} J \otimes \mathbb{Q}$ are the Lie subalgebras of $D_{*}(H) \otimes \mathbb{Q}$ generated by elements of degree 1 .

The exact same considerations apply to the lower central series filtration of $\mathcal{H}_{g}$ and we obtain a Lie algebra homomorphism

$$
j^{H}: \mathcal{G}_{*}^{l}\left(\mathcal{T H}_{g}\right) \rightarrow \mathcal{G}_{*}^{w}\left(\mathcal{H}_{g}\right) \cong D_{*}(H)
$$

The image of $j^{H} \otimes \mathbb{Q}$ is again the subalgebra generated by degree 1 elements, which is the same as $\operatorname{Im} J \otimes \mathbb{Q}$, and so we conclude that $j^{H}$ is not onto.

\subsection{Finite-type invariants of $\mathcal{H}_{g}$}

There have been several proposals for a theory of finite-type invariants for general 3-manifolds, extending Ohtsuki's theory [Oh] for homology 3-spheresperhaps the first given in Cochran-Melvin $[\mathrm{CM}]$. We will use, however, the particular version proposed independently by M. Goussarov [Go],[GGP] and K. Habiro [H]. We will show that $\eta_{k}$ and a homomorphism $\beta: \mathcal{T} \mathcal{H}_{g} \rightarrow V$ defined by Birman-Craggs homomorphisms [BC], where $V$ is a vector space over $Z / 2$, make up the universal multiplicative homology bordism invariant of type $k$ on the class of homology cylinders for any $k \geq 1$. It is to be emphasized that we make use of results announced in [H] but, for which no proofs have yet appeared.

We give a brief summary of the Goussarov-Habiro theory, and refer the reader to $[\mathrm{GGP}],[\mathrm{H}],[\mathrm{Ha}]$ for details. Let $G$ be a unitrivalent graph whose trivalent vertices are equipped with a cyclic ordering of its incident edges and whose univalent vertices are decorated with an element of an abelian group $H$. We also insist that each component of $G$ have at least one trivalent vertex. We refer to such a graph as an $H$-graph and define the degree of $G$ to be the number of trivalent vertices. If $M$ is a 3 -manifold and $H=H_{1}(M)$, then a clasper (using the terminology of $[\mathrm{H}]$ - clover in the terminology of [GGP]) in $M$ associated to $G$ is a framed link $C$ in $M$ obtained in the following way. Associate to each trivalent vertex of $G$ a copy of the Borromean rings, in disjoint balls of $M$, each component associated to an end of an edge incident to that vertex (even if two of those ends are just opposite ends of the same edge) and given the 0 -framing. Associate to each univalent vertex a framed knot in $M$, disjoint from the balls, and representing the element of $H$ labeling that vertex. These 
are the leaves of the clasper. Finally for each edge of $G$ introduce a simple clasp between the knots associated to the two ends of that edge. The construction of a clasper $C$ from an $H$-graph $G$ involves a choice of framed imbedding into $M$ of the graph obtained from $G$ by attaching circles to the univalent vertices. See Figure 3 for a typical example. If $G$ has degree $n$ we say $C$ is
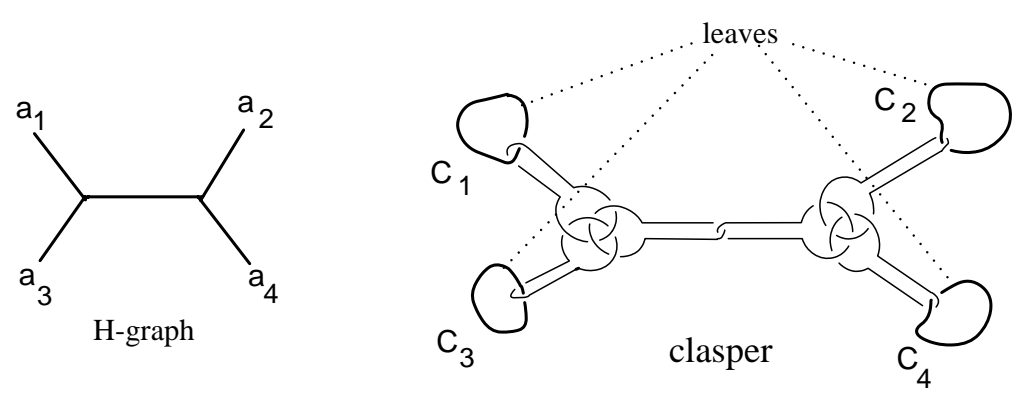

Figure 3: An H-graph and an associated clasper, where $C_{i}$ represents $a_{i}$

an $n$-clasper. Surgery on a clasper produces a new manifold $M^{\prime}=M_{C}$ with isomorphic homology and torsion pairing. We will refer to surgery on an $n$ clasper as an $n$-surgery. Thus if $M$ is a homology cylinder of genus $g$ so is $M^{\prime}$. It is easy to see that the associated automorphisms of $H_{1}\left(\Sigma_{g, 1}\right)$ associated to $M$ and $M^{\prime}$ are the same. Matveev [Ma] has proved the converse: If $M, M^{\prime}$ have isomorphic homology and torsion pairing then there is a clasper $C$ in $M$ such that $M^{\prime}=M_{C}$. We define the relation of $A_{k}$-equivalence (in the terminology of $[\mathrm{H}])$ to be generated by the following elementary move: $M \sim_{k} M$ if $M^{\prime}$ is diffeomorphic to $M_{C}$, for some connected $k$-clasper $C$. According to [H], the set of $A_{k}$-equivalence classes in $\mathcal{C}_{g}$ is a group (finitely-generated and nilpotent) under the stacking multiplication. Let $\mathcal{F}_{k}\left(\mathcal{C}_{g}\right)$ denote the subsemigroup of $\mathcal{C}_{g}$ consisting of homology cylinders $A_{k}$-equivalent to the trivial one $I \times \Sigma_{g, 1}$ and let $\mathcal{G}_{k}\left(\mathcal{C}_{g}\right)=\mathcal{F}_{k}\left(\mathcal{C}_{g}\right) / A_{k+1}$

Habiro defines $\mathcal{A}_{k}(H)$, for $k>1$, to be the abelian group generated by connected $H$-graphs of degree $k$ with an extra structure of a total ordering of the univalent vertices, subject to the AS and IHX relations, multilinearity of the labels and an STU-like relation(see $[\mathrm{H}]) . \mathcal{A}_{1}(H)$ is defined explicitly to be $\Lambda^{3} H \oplus V$, where $V=\Lambda^{2} H_{2} \oplus H_{2} \oplus \mathbb{Z} / 2$, where $H_{2}=H \otimes \mathbb{Z} / 2$. Habiro constructs an epimorphism $\mathcal{A}_{k}(H) \rightarrow \mathcal{G}_{k}\left(\mathcal{C}_{g}\right)$, which can be defined for $k>1$ by clasper surgery on $I \times \Sigma_{g, 1}$, in which the ordering of the univalent vertices is used to stack the imbeddings (with horizontal framings) of the leaves representing the elements of $H=H_{1}\left(\Sigma_{g, 1}\right)$ decorating those vertices. Habiro conjectures that 
this epimorphism is an isomorphism and claims that it is so for $k=1$ and, for $k>1$, induces an isomorphism $\mathcal{A}_{k}(H) \otimes \mathbb{Q} \cong \mathcal{G}_{k}\left(\mathcal{C}_{g}\right) \otimes \mathbb{Q}$. See [Ha] for an alternative construction for homology handlebodies.

Let $\mathcal{A}_{k}^{\circ}(H)$ denote, for $k>1$, the subgroup of $\mathcal{A}_{k}(H)$ generated by connected $H$-graphs with non-zero first Betti number, i.e. with a non-trivial cycle. The quotient $A_{k}^{t}(H)=\mathcal{A}_{k}(H) / \mathcal{A}_{k}^{\circ}(H)$ is generated by $H$-trees. Note that the ordering of the univalent vertices in $\mathcal{A}_{k}^{t}(H)$ does not matter, because of the STU-relation, and so this structure can be ignored. Set $\mathcal{A}_{1}^{t}(H)=\mathcal{A}_{1}(H)$.

We recall a fact known to the experts (see also [Ha]).

Proposition 2.2 $\mathcal{A}_{k}^{t}(H) \cong \mathrm{D}_{k}(H)$ for $k>1$. $\mathcal{A}_{1}^{t}(H) \rightarrow \mathrm{D}_{1}(H) \cong \Lambda^{3} H$.

Outline of proof. If $k=1$, this was already pointed out by Johnson [J]. Now suppose $k>1$. Let $T$ be an $H$-tree of degree $k$. If we choose any univalent vertex to be the root of $T$, then we can associate to this rooted $H$ tree an element in $H \otimes L_{k+1}(H)$. For example, if $T$ is the $H$-graph in Figure 3 , with the counter-clockwise orientation at each vertex and root at $a_{1}$, then the associated element would be $a_{1} \otimes\left[\alpha_{3},\left[\alpha_{4}, \alpha_{3}\right]\right]$, where $\alpha_{i}$ is a lift of $a_{i}$ into $\pi_{1}\left(\Sigma_{g, 1}\right)$. Now we associate to $T$ the sum of the associated elements of $H \otimes L_{k+1}(H)$ as the roots range over all the univalent vertices of $T$. It is not hard to see that this element actually lies in $\mathrm{D}_{k}(H)$ and that, in fact, $\mathrm{D}_{k}(H)$ is generated by the elements associated to all possible $H$-trees of degree $k$.

To bring homology bordism into the picture we need the following theorem.

Theorem 2 Let $M$ be a 3-manifold and $G$ a connected $H$-graph, where $H=H_{1}(M)$, with at least one non-trivial cycle. If $C$ is any clasper associated to $G$, then $M_{C}$ is homology bordant to $M$.

Remark 2.3 As an immediate consequence of this theorem we conclude that if $L \subseteq M$ is a link in a 3 -manifold and $C$ is such a clasper in $M-L$ then the links $(M, L)$ and $\left(M_{C}, L\right)$ are homology concordant, i.e. there is a homology bordism $V$ between $M$ and $M_{C}$ and a proper imbedding $I \times L \subseteq V$ such that $0 \times L=L \subseteq M$ and $1 \times L=L \subseteq M_{C}$. Compare this to Theorem 2.9 of [Ha] which shows that, in the case of $M$ a homology ball, the Milnor $\bar{\mu}$-invariants of $L \subseteq M$ and $L \subseteq M_{C}$ coincide.

Remark 2.4 A more delicate argument will strengthen Remark 2.3 as follows. If the clasper $C$ is strict in the sense of [H], i.e. the leaves of $C$ bound disks which 
are disjoint from each other and the rest of $C$ except for a single intersection point with the companion component of $C$ (but will generally intersect $L$ ), then $\left(M_{C}, L\right)$ is concordant to $(M, L)$, i.e. there is an imbedding $I \times L \subseteq I \times M$ so that $(0 \times M, 0 \times L)$ is diffeomorphic to $(M, L)$ and $(1 \times M, 1 \times L)$ is diffeomorphic to $\left(M_{C}, L\right)$.

To prove Theorem 2 we need the following Lemma.

Lemma 2.5 Let $M$ be a 3-manifold and $L \subseteq M$ a framed link. Suppose that $L=L^{\prime} \cup L^{\prime \prime}$, where $L^{\prime}$ and $L^{\prime \prime}$ have the same number of components and

(a) $L^{\prime}$ is a trivial link bounding disjoint disks $D$,

(b) $L^{\prime}$ is 0 -framed,

(c) the matrix of intersection numbers of the components of $D$ with those of $L^{\prime \prime}$ is non-singular.

Then $M_{L}$ is homology bordant to $M$.

Proof We construct a manifold $V$ from $I \times M$ by adjoining handles to $0 \times M$ along the framed link $L^{\prime \prime}$ and by removing tubular neighborhoods of properly imbedded disjoint disks $D^{\prime}$ in $I \times M$ obtained by pushing $0 \times \operatorname{Int} D$ into the interior of $I \times M$. Then $\partial V=M_{L}-(1 \times M)$, so it only remains to observe that the pair $(V, 1 \times M)$ is acyclic.

Let $W=(I \times M)-D^{\prime}$. It is easy to see that the only non-zero homology group of $(W, 1 \times M)$ is $H_{1}(W, 1 \times M)$, which is freely generated by the meridians of $D^{\prime}$. By considering the triple $(V, W, 1 \times M)$ we find there is an exact sequence:

$$
0 \rightarrow H_{2}(V, 1 \times M) \rightarrow H_{2}(V, W) \rightarrow H_{1}(W, 1 \times M) \rightarrow H_{1}(V, 1 \times M) \rightarrow 0
$$

Now $H_{2}(V, W)$ is the only non-zero homology group of $(V, W)$ and it is freely generated by the disks adjoined along $L^{\prime \prime}$. Since the homomorphism $H_{2}(V, W)$ $\rightarrow H_{1}(W, 1 \times M)$ is represented by the matrix of intersection numbers of the components of $D^{\prime}$ with those of $I \times L^{\prime \prime}$, it follows that $(V, 1 \times M)$ is acyclic if and only if (c) is satisfied.

Proof of Theorem 2 Recall that for each edge of $G$ there are two components of $C$, one at each end of the edge (see Figure 3). We will call them companion components. We will construct $C^{\prime}$ and $C^{\prime \prime}$ by assigning, for each edge of $G$, one of the associated companions to $C^{\prime}$ and the other to $C^{\prime \prime}$. This choice can be represented by an orientation of the edge pointing from the end associated to the companion in $C^{\prime}$ toward the end associated to $C^{\prime \prime}$. Our aim will be to make these choices satisfy: 
(i) An edge with a univalent vertex ( a leaf of $G$ ) is oriented toward the leaf, i.e. outward (thus the leaves of $C$ will all belong to $C^{\prime \prime}$ ),

(ii) No trivalent vertex is a source, i.e. not all the incident edges are oriented away from the vertex.

We will see that these conditions can be satisfied if and only if $G$ is not a tree. But for now note that if these conditions are satisfied then the decomposition of $C$ will satisfy conditions (a)-(c) of Lemma 2.5. Since the three components of $C$ associated to any trivalent vertex are a Borromean rings, any two of the components bound disjoint disks. Thus we can choose disjoint disks $D$ bounded by each component of $C^{\prime}$, the disks from components associated to different vertices will be disjoint. The only problem would be if these components were associated to the same edge, but this is ruled out. Now each disk from a component of $C^{\prime}$ will intersect the companion component of $C$ once - the only other intersections will be with one of the components of $C^{\prime \prime}$ which is associated to the same trivalent vertex, but the intersection number will be 0 . Thus the intersection matrix of the components of $D$ with those of $C^{\prime \prime}$ will be the identity matrix. See Figure 4 for an example.
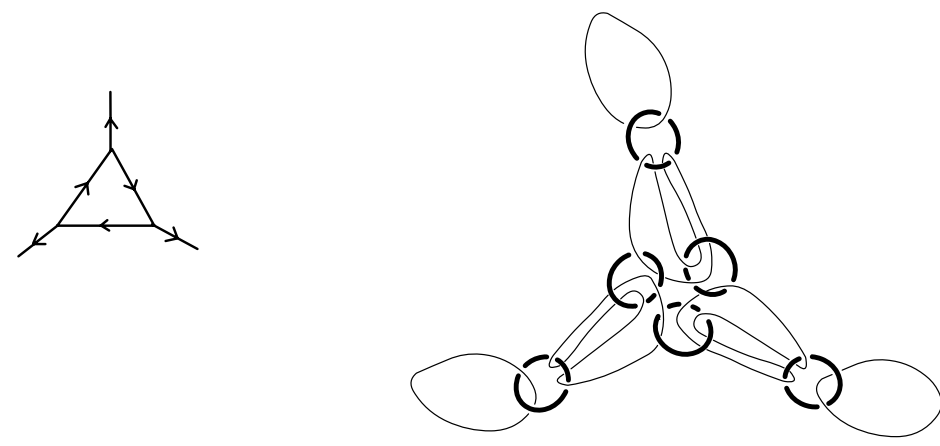

Figure 4: An oriented graph on the left and a corresponding clasper $C$ on the right. The bold components define $C^{\prime}$.

Lemma 2.6 Suppose that $T$ is a unitrivalent tree, with an orientation prescribed for each leaf edge, not all outward. Then we can extend this to an orientation of all the edges of $T$ which satisfies (ii).

Proof A similar fact is proved in [Ha]. Choose one of the leaves $e$ of $T$ which is oriented inward. Now orient every edge of $T$ which is not a leaf so that it points away from $e$, i.e. if we travel along any non-singular edge path which begins at $e$ and ends at a non-leaf, then the orientations of all the edges in the 
path point in the direction of travel. Then it is clear that any trivalent vertex will have at least one of its incident edges oriented toward that vertex. Thus (ii) will be satisfied whatever the orientations of the other leaves. See Figure 5 for an example.

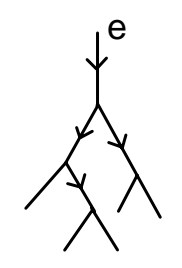

Figure 5

We can now complete the proof of Theorem 2. Since $G$ has a cycle we can make one or more cuts in edges of $G$ to create a tree. Each edge of $G$ which is cut will create two new leaves in $T$. We now choose arbitrary orientations of each cut edge of $G$, which will induce orientations of the new leaf edges of $T$. Note that one of each pair of new leaves will be oriented inwards. Thus the outward orientations of the leaves of $G$ together with these orientations of the new leaves of $T$ provides orientations of all the leaves of $T$ which satisfies the hypothesis of Lemma 2.6. Applying this lemma gives an orientation of $T$ satisfying (ii). But now we can glue the cut edges back together and we get an orientation of $G$ satisfying (i) and (ii), thus proving the Theorem. See Figure 6 for an example.
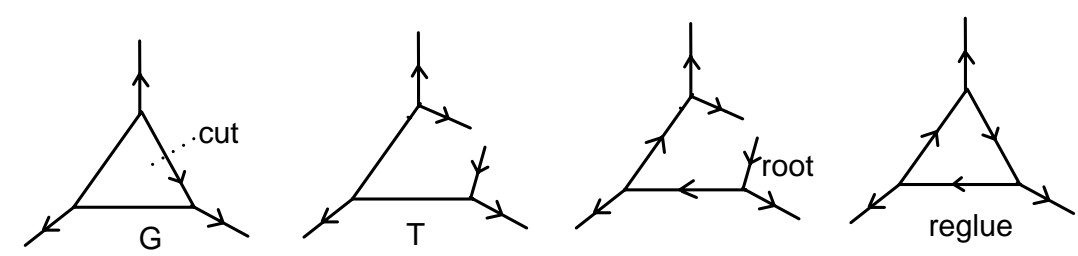

Figure 6

Let's define a filtration of $\mathcal{H}_{g}$ by $\mathcal{F}_{k}^{Y}\left(\mathcal{H}_{g}\right)=\operatorname{Im}\left(\mathcal{F}_{k}\left(\mathcal{C}_{g}\right) \rightarrow \mathcal{H}_{g}\right)$, where we consider the restriction of the quotient map $\mathcal{C}_{g} \rightarrow \mathcal{H}_{g}$. By [Ma], $\mathcal{F}_{1}^{Y}\left(\mathcal{H}_{g}\right)=$ $\mathcal{F}_{1}^{w}\left(\mathcal{H}_{g}\right)=\mathcal{T H}_{g}$

Theorem $3 \mathcal{F}_{k}^{Y}\left(\mathcal{H}_{g}\right) \subseteq \mathcal{F}_{k}^{w}\left(\mathcal{H}_{g}\right)$, thus inducing a map $\mathcal{G}_{k}^{Y}\left(\mathcal{H}_{g}\right) \rightarrow \mathcal{G}_{k}^{w}\left(\mathcal{H}_{g}\right)$ 
and a commutative diagram:

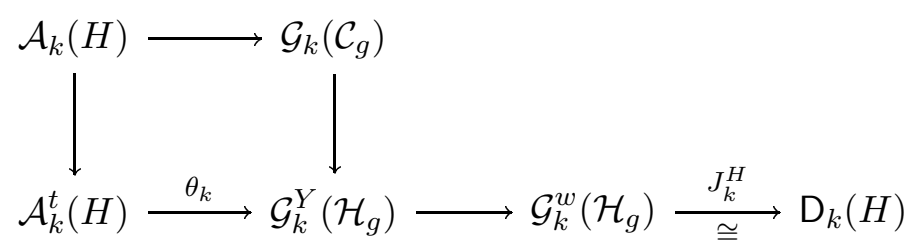

$\theta_{k}$ is an isomorphism for all $k$ and the composition $\mathcal{A}_{k}^{t}(H) \rightarrow \mathcal{G}_{k}^{Y}\left(\mathcal{H}_{g}\right) \rightarrow$ $\mathcal{G}_{k}^{w}\left(\mathcal{H}_{g}\right) \rightarrow \mathrm{D}_{k}(H)$ is the epimorphism (isomorphism if $k>1$ ) of Proposition 2.2.

Proof First note that it follows from Theorem 2 that the composition $\mathcal{A}_{k}(H)$ $\rightarrow \mathcal{G}_{k}\left(\mathcal{C}_{g}\right) \rightarrow \mathcal{G}_{k}^{Y}\left(\mathcal{H}_{g}\right)$ factors through $\mathcal{A}_{k}^{t}(H)$. This yields the commutative diagram (3), assuming for the moment that $\mathcal{F}_{k}^{Y}\left(\mathcal{H}_{g}\right) \subseteq \mathcal{F}_{k}^{w}\left(\mathcal{H}_{g}\right)$.

To see that $\mathcal{F}_{k}^{Y}\left(\mathcal{H}_{g}\right) \subseteq \mathcal{F}_{k}^{w}\left(\mathcal{H}_{g}\right)$ for $k>1$, and, at the same time, identify the composition of the maps in the bottom line of the diagram, we first consider a $k$-clasper $C$ in $I \times \Sigma_{g, 1}$ associated to a connected $H$-tree $T$. If we choose a root of $T$ and the element of $H \otimes L_{k+1}(H)$ associated to this rooted $H$ tree is $\alpha \otimes \gamma$, then, by a sequence of Kirby moves, we can convert $C$ into a 2-component link $\left(l, l^{\prime}\right)$, where $l$ is the leaf of $C$ corresponding to the root, and so represents $\alpha \in \pi / \pi_{2}$, and $l^{\prime}$ represents $\gamma \in \pi_{k+1} \bmod \pi_{k+2}$. For example, Figure 7 explains this for a rooted $H$-tree of degree 1 .
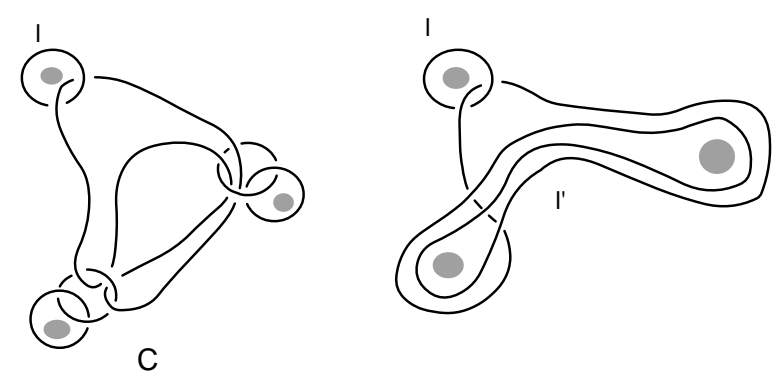

Figure 7: A clasper $C$ on the left, and the Kirby-equivalent link on the right

Now suppose $M=\left(I \times \Sigma_{g, 1}\right)_{C}$ and $\xi \in \pi_{1}\left(\Sigma_{+}\right)$. To compute $\eta_{k}(M) \cdot \xi$ we push a curve $K$ representing $\xi$ down $I \times \Sigma_{g, 1}$ and observe how it changes every time we cross $C$. When it crosses the leaf $l$, the effect may be computed by using the Kirby-equivalent link $l \cup l^{\prime}$ instead of $C$. But then it is not hard to see that the effect is to add a copy of $l^{\prime}$ to $K$. Thus the contribution of all the crossings of $K$ with the leaf $l$ is $\langle\xi, \alpha>\gamma$, where $<,>$ denotes the 
homological intersection number in $\Sigma_{g, 1}$. Under the canonical isomorphism $\operatorname{Hom}\left(H, L_{k+1}(H)\right) \cong H \otimes L_{k+1}(H)$ this corresponds to $\alpha \otimes \gamma$. The total change in $\xi$ is then the sum of these contributions over all the univalent vertices of $T$, which is exactly the element of $\mathrm{D}_{k}(H)$ corresponding to $T$. This shows that $\mathcal{F}_{k}^{Y}\left(\mathcal{H}_{g}\right) \subseteq \mathcal{F}_{k}^{w}\left(\mathcal{H}_{g}\right)$, at least for the elements represented by trees in $\mathcal{F}_{k}^{Y}\left(\mathcal{H}_{g}\right)$, and that the composition $\mathcal{A}_{k}(H) \rightarrow \mathcal{G}_{k}\left(\mathcal{C}_{g}\right) \rightarrow \mathcal{G}_{k}^{Y}\left(\mathcal{H}_{g}\right) \rightarrow \mathcal{G}_{k}^{w}\left(\mathcal{H}_{g}\right) \rightarrow$ $\mathrm{D}_{k}(H)$ associates to any $H$-tree the corresponding element of $\mathrm{D}_{k}(H)$ under the isomorphism of Proposition 2.2. The proof of the commutativity of diagram (3) is completed by noting that if $G$ is any $H$-graph with a cycle (and an ordering of its univalent vertices), then we can cut open some edges to create a tree $T$, where the new vertices are labeled by $0 \in H$. Now a clasper representing $G$ is also a clasper representing $T$ and the above argument applies. Since some of the labels are 0 , the image in $\mathrm{D}_{k}(H)$ is 0 .

To prove that $\theta_{k}$ is an isomorphism first note that the map $\mathcal{A}_{k}^{t}(H) \rightarrow \mathcal{G}_{k}^{Y}\left(\mathcal{H}_{g}\right)$ is onto, since $\mathcal{A}_{k}(H) \rightarrow \mathcal{G}_{k}\left(\mathcal{C}_{g}\right)$ is onto. So if $k>1$ it must also be one-one, since the composition to $\mathrm{D}_{k}(H)$ is an isomorphism. Thus all the maps in the bottom row must be isomorphisms.

To deal more completely with $k=1$ we must consider some additional $\mathbb{Z} / 2$ invariants on $\mathcal{H}_{g}$ - or rather on $\mathcal{T H}_{g}=\mathcal{F}_{1}^{Y}\left(\mathcal{H}_{g}\right)=\mathcal{F}_{1}^{w}\left(\mathcal{H}_{g}\right)$. Recall the BirmanCraggs homomorphisms on the classic Torelli group $T_{g}$ from $[\mathrm{BC}]$. To define these first choose an imbedding $i: \Sigma_{g} \subseteq S^{3}$. Then if $h \in T_{g}$ we can cut $S^{3}$ along $i\left(\Sigma_{g}\right)$ and reglue, using $h$, to obtain a homology sphere $\Sigma_{h}$. We then define $\beta_{i}(h)$ to be the Rochlin invariant of $\Sigma_{h}$. It is clear that this makes perfectly good sense if we substitute a homology cylinder in $\mathcal{T H}_{g}$ for $h$ and the result depends only on its homology bordism class. Thus we obtain homomorphisms $\beta_{i}: \mathcal{T H}_{g} \rightarrow \mathbb{Z} / 2$.

Johnson shows that one can choose a number of $\left\{\beta_{i}\right\}$ exactly equal to the dimension of the vector space $V$, thereby defining a homomorphism $\beta: T_{g} \rightarrow$ $V$, which determine all the Birman-Craggs homomorphisms, and he then shows that the combined homomorphism $H_{1}\left(T_{g}\right) \rightarrow \Lambda^{3} H \oplus V$ is an isomorphism.

Now consider the corresponding $\beta: \mathcal{T H}_{g} \rightarrow V$. We claim that $\beta\left(\mathcal{F}_{2}^{Y}\left(\mathcal{H}_{g}\right)\right)=0$ and so we get an induced $\beta: \mathcal{G}_{1}^{Y}\left(\mathcal{H}_{g}\right) \rightarrow V$. As is pointed out in $[\mathrm{H}]$ and [GGP], surgery on a clasper of degree $n$ is the same as cutting and pasting along some imbedded surface using an element of the $n$-th lower central series term $\left(T_{g}\right)_{n}$ of the Torelli group. Thus a Birman-Craggs homomorphism on a homology cylinder in $\mathcal{F}_{n}^{Y}\left(\mathcal{H}_{g}\right)$ takes the same value as some Birman-Craggs homomorphism on some element of $\left(T_{g}\right)_{n}$, which is always zero if $n \geq 2$. 
Let us now consider the combined homomorphism $\mathcal{G}_{1}^{Y}\left(\mathcal{H}_{g}\right) \rightarrow \mathcal{G}_{1}^{w}\left(\mathcal{H}_{g}\right) \oplus V \cong$ $\Lambda^{3} H \oplus V$, which must be an epimorphism since it is when restricted to $T_{g}$. Since we also have Habiro's epimorphism $\Lambda^{3} H \oplus V \cong \mathcal{A}_{1}^{t}(H) \rightarrow \mathcal{G}_{1}^{Y}\left(\mathcal{H}_{g}\right)$, it follows that both of these epimorphisms are actually isomorphisms.

To summarize, we have:

Corollary 2.7 (1) $\mathcal{G}_{1}^{Y}\left(\mathcal{H}_{g}\right) \cong \mathcal{G}_{1}^{w}\left(\mathcal{H}_{g}\right) \oplus V \cong \Lambda^{3} H \oplus V$,

(2) $\mathcal{G}_{k}^{Y}\left(\mathcal{H}_{g}\right) \cong \mathcal{G}_{k}^{w}\left(\mathcal{H}_{g}\right)$ for $k>1$,

(3) $\mathcal{F}_{k}^{Y}\left(\mathcal{H}_{g}\right)=\mathcal{F}_{k}^{w}\left(\mathcal{H}_{g}\right) \cap \operatorname{Ker} \beta$ for $k>1$.

Proof (1) and (2) are proved above. To prove (3) first consider $k=2$. Suppose that $M \in \mathcal{F}_{2}^{w}\left(\mathcal{H}_{g}\right) \subseteq \mathcal{F}_{1}^{w}\left(\mathcal{H}_{g}\right)=\mathcal{F}_{1}^{Y}\left(\mathcal{H}_{g}\right)$ and $\beta(M)=0$. Then $M \rightarrow 0 \in$ $\mathcal{G}_{1}^{w}\left(\mathcal{H}_{g}\right) \oplus V \cong \mathcal{G}_{1}^{Y}\left(\mathcal{H}_{g}\right)$ and so $M \in \mathcal{F}_{2}^{Y}\left(\mathcal{H}_{g}\right)$. For $k \geq 3$ we proceed by induction, using the following commutative diagram.

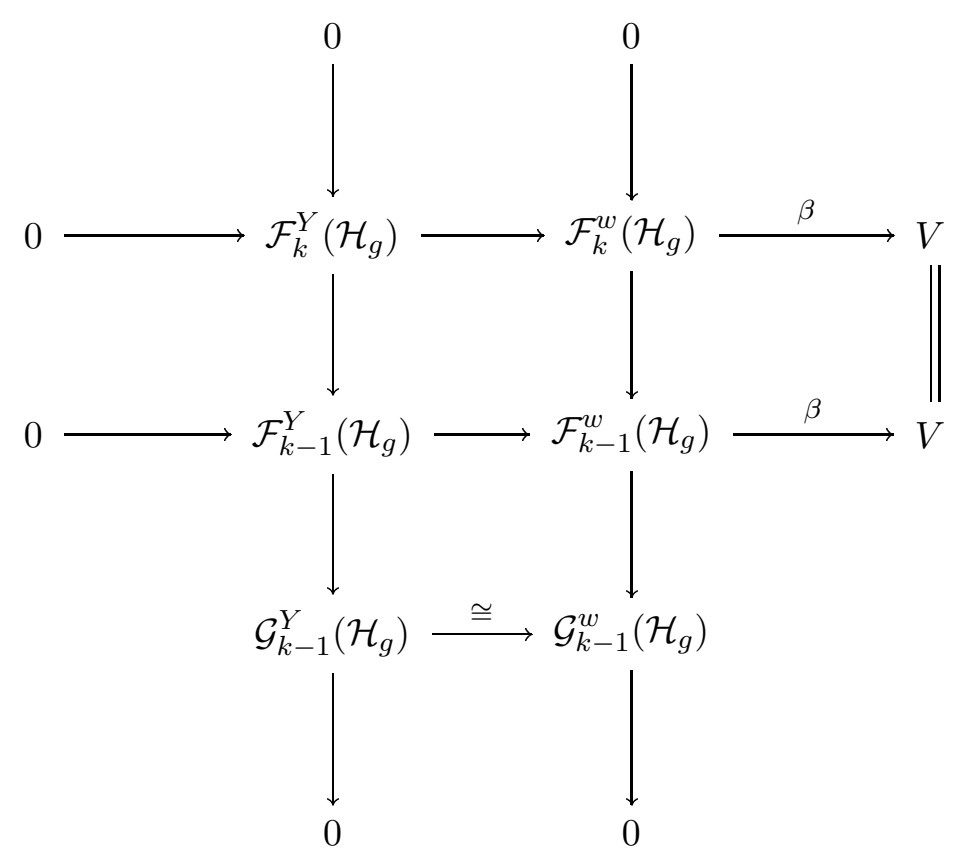

The columns are exact and, by induction, the middle row is exact. It then follows that the top row is exact.

Suppose that $\phi$ is a multiplicative invariant of homology cylinders of genus $g$, i.e. a homomorphism $\phi: \mathcal{C}_{g} \rightarrow G$ for some group $G$. We will say that $\phi$ is of finite type if $\phi\left(\mathcal{F}_{k+1}\left(\mathcal{C}_{g}\right)\right)=1$ for some $k$, and is of type $k$ for the minimum 
such value. This is actually a weaker definition than the usual notion of finite type.

For example $\eta_{k}$ is an invariant of type $k$, as is $\beta$, although it is only defined on $\mathcal{T} \mathcal{H}_{g}$. The following corollary asserts that $\eta_{k}$ and $\beta$ are the universal homology bordism invariant of type $k$.

Corollary 2.8 Suppose $\phi$ is a multiplicative homology bordism invariant of type $k$. If $M, N$ are two homology cylinders such that $\eta_{k}(M)=\eta_{k}(N)$ and $\beta\left(M \cdot N^{-1}\right)=0$, then $\phi(M)=\phi(N)$.

Proof By Corollary $2.7 M \cdot N^{-1} \in \mathcal{F}_{k+1}^{Y}\left(\mathcal{H}_{g}\right)$ and so $\phi\left(M \cdot N^{-1}\right)=1$.

\section{$3 \quad$ String links and homology cylinders}

\subsection{String links}

Let $\mathcal{P}_{g}$ denote the group of pure braids with $g$ strands. Let $\mathcal{S}_{g}$ denote the group of concordance classes of string links with $g$ strands in a homology 3 ball whose boundary is identified with $\partial\left(I \times D^{2}\right)$. Two string links $S_{1}, S_{2}$ in homology 3 -balls $B_{1}, B_{2}$, respectively, are concordant if there is a homology 4-ball $B$ whose boundary is identified with $B_{1} \cup B_{2}$ (with their boundaries identified), and a concordance between $S_{1}$ and $S_{2}$ imbedded in $B$. There is an obvious homomorphism $\mathcal{P}_{g} \rightarrow \mathcal{S}_{g}$. Recall the theorem of Artin which states that the map $\mathcal{P}_{g} \rightarrow \operatorname{Aut}\left(F^{\prime}\right)$-where $F^{\prime}$ is the free group with basis $\left\{y_{1}, \ldots, y_{g}\right\}$ identified with $\pi_{1}\left(D_{g}\right)$, and $D_{g}$ is the 2-disk with $g$ holes-is injective with image $\operatorname{Aut}_{1}\left(F^{\prime}\right)$. Aut $\left(F^{\prime}\right)$ is the subgroup of $\operatorname{Aut}\left(F^{\prime}\right)$ consisting of all automorphisms $h$ such that $h\left(y_{i}\right)=\lambda_{i}^{-1} y_{i} \lambda_{i}$, for some choice of $\lambda_{i}$ and $h\left(y_{1} \cdots y_{g}\right)=y_{1} \cdots y_{g}$. Note that $\lambda_{i}$ is uniquely determined if we specify that the exponent sum of $y_{i}$ in $\lambda_{i}$ is 0 .

The Milnor $\bar{\mu}$-invariants can be formulated as a sequence of homomorphisms $\bar{\mu}_{k}: \mathcal{S}_{g} \rightarrow \operatorname{Aut}_{1}\left(F^{\prime} / F_{k+2}^{\prime}\right), k \geq 1$, where $\operatorname{Aut}_{1}\left(F^{\prime} / F_{q}^{\prime}\right)$ consists of all automorphisms $h$ satisfying $h\left(y_{i}\right)=\lambda_{i}^{-1} y_{i} \lambda_{i}$ for some $\lambda_{i} \in F^{\prime} / F_{q-1}^{\prime}$ and satisfying the equation

$$
h\left(y_{1} \cdots y_{g}\right)=y_{1} \cdots y_{g}
$$

(see [HL]). Note now that $\lambda_{i}$ is uniquely determined by $h$ up to left multiplication by a power of $y_{i}$. One consequence of the existence of the $\bar{\mu}_{k}$ is that 
the map $\mathcal{P}_{g} \rightarrow \mathcal{S}_{g}$ is injective. It is known that $\bar{\mu}_{k}$ is onto (see e.g. [HL]). If we define $\mathcal{S}_{g}[k]=\operatorname{Ker} \bar{\mu}_{k}$ and $\mathcal{S}_{g}[0]=\mathcal{S}_{g}$, then we have an isomorphism $\mathcal{S}_{g}[k] / \mathcal{S}_{g}[k+1] \cong \mathrm{D}_{k}\left(H^{\prime}\right)$, for $k \geq 0$, where $H^{\prime}=H_{1}\left(D_{g}\right)$. This isomorphism is defined by $\sigma \rightarrow \sum_{i} y_{i} \otimes \lambda_{i}$, for $\sigma \in \mathcal{S}_{g}[k]$, where $\lambda_{i} \in F_{k+1} / F_{k+2}$ are determined by $\mu_{k+1}$. Note that $\mathcal{S}_{g}[\infty]=\bigcap_{k} \mathcal{S}_{g}[k]$ is non-trivial since, for example, it contains the knot concordance group.

If $\mathcal{S}_{g}^{0}$ denotes the standard string link concordance group, i.e. string links in $I \times D^{2}$ and concordances in $I \times\left(I \times D^{2}\right)$, then we have natural maps $\mathcal{P}_{g} \subseteq$ $\mathcal{S}_{g}^{0} \rightarrow \mathcal{S}_{g}$.

Question 1 Is $\mathcal{S}_{g}^{0} \rightarrow \mathcal{S}_{g}$ injective? Since the realization theorem of [HL] produces standard string links the induced map $\mathcal{S}_{g}^{0} / \mathcal{S}_{g}^{0}[\infty] \rightarrow \mathcal{S}_{g} / \mathcal{S}_{g}[\infty]$ is an isomorphism.

If $\theta$ denotes the group of homology bordism classes of closed homology 3spheres, then we have an obvious injection $\theta \subseteq \mathcal{S}_{g}$, defined by connected sum with the trivial string link in $I \times D^{2}$, whose image is a central subgroup of $\mathcal{S}_{g}$, lying in $\mathcal{S}_{g}[\infty]$, and a retraction $\mathcal{S}_{g} \rightarrow \theta$.

Question 2 Is the combined map $\theta \times \mathcal{S}_{g}^{0} \rightarrow \mathcal{S}_{g}$ an isomorphism?

We will need to consider the framed versions. Let $\mathcal{P}_{g}^{\text {fr }}$ and $\mathcal{S}_{g}^{\text {fr }}$ denote the groups of framed pure braids on $g$ strands and concordance classes of framed string links with $g$ strands. Note that $\mathcal{P}_{g}^{\text {fr }} \cong \mathcal{P}_{g} \times \mathbb{Z}^{g}$ and $\mathcal{S}_{g}^{\text {fr }} \cong \mathcal{S}_{g} \times \mathbb{Z}^{g}$. We have an isomorphism $\mu: \mathcal{P}_{g}^{\mathrm{fr}} \rightarrow \mathcal{A}_{1}\left(F^{\prime}\right)$ and homomorphisms $\mu_{k}: \mathcal{S}_{g}^{\mathrm{fr}} \rightarrow \mathcal{A}_{1}\left(F^{\prime} / F_{k+1}^{\prime}\right)$ for $k \geq 1$. $\mathcal{A}_{1}\left(F^{\prime}\right)$ (resp. $\left.\mathcal{A}_{1}\left(F^{\prime} / F_{q}^{\prime}\right)\right)$ consists of sequences $\lambda=\left(\lambda_{1}, \ldots, \lambda_{g}\right)$ of elements $\lambda_{i} \in F^{\prime}$ (resp. $F^{\prime} / F_{q}^{\prime}$ ) which satisfy the following

(i) The map $y_{i} \rightarrow \lambda_{i}^{-1} y_{i} \lambda_{i}$ defines an automorphism $\phi_{\lambda}$ of $F^{\prime}$ (resp. $F^{\prime} / F_{q+1}^{\prime}$ )

(ii) $\phi_{\lambda}\left(y_{i} \cdots y_{g}\right)=y_{1} \cdots y_{g}$.

The multiplication in $\mathcal{A}_{1}\left(F^{\prime}\right), \mathcal{A}_{1}\left(F^{\prime} / F_{q}^{\prime}\right)$ is defined by $(\lambda \mu)_{i}=\lambda_{i} \phi_{\lambda}\left(\mu_{i}\right)$. Then $\lambda \rightarrow \phi_{\lambda}$ defines epimorphisms with kernel $\mathbb{Z}^{g}$. Note that we have the commutative diagram

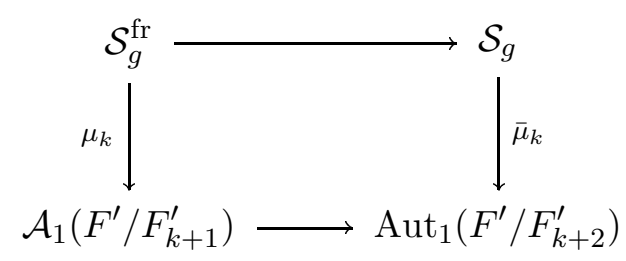

We define the filtration $\mathcal{S}_{g}^{\mathrm{fr}}[k]=\operatorname{Ker} \mu_{k}$. 


\subsection{Relating string links and homology cylinders}

Recall the imbedding $\Phi: \mathcal{P}_{g}^{\mathrm{fr}} \rightarrow \Gamma_{g, 1}$, defined by [O] and studied in [L, Section 2.2]. There is a commutative diagram

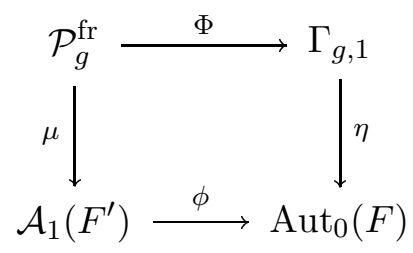

where $\phi$ is defined by

$$
\begin{aligned}
& \phi\left(\left(\lambda_{i}\right)\right) \cdot y_{i}=\lambda_{i}^{-1} y_{i} \lambda_{i} \\
& \phi\left(\left(\lambda_{i}\right)\right) \cdot x_{i}=x_{i} \lambda_{i}
\end{aligned}
$$

Since $\mu$ and $\eta$ are isomorphisms and $\phi$ is injective, it follows that $\Phi$ is injective.

We now extend $\Phi$ to an imbedding $\hat{\Phi}: \mathcal{S}_{g}^{\text {fr }} \rightarrow \mathcal{H}_{g}$. Choose an imbedding $D_{g} \subseteq \Sigma_{g, 1}$ where the meridians of the holes in $D_{g}$, which correspond to the generators $y_{i} \in F^{\prime}$, are mapped to the meridians of the handles of $\Sigma_{g, 1}$ which correspond to the generators $y_{i} \in F$-see Figure 1 . Suppose $S$ is a framed string link with $g$ strands in a homology 3-ball $B$ and $C$ is the complement of an open tubular neighborhood of $S$ in $B$. Then there is a canonical identification of $\partial C$ with $\partial\left(I \times D_{g}\right)$. Now we create a homology cylinder by taking $I \times \Sigma_{g, 1}$, with $\Sigma^{-}=0 \times \Sigma_{g, 1}, \Sigma^{+}=1 \times \Sigma_{g, 1}$, removing $I \times D_{g}$ and replacing it with $C$, using the canonical identification of the boundaries. We take this homology cylinder to represent $\hat{\Phi}(S)$.

It is clear that $\hat{\Phi}$ is a well-defined homomorphism and extends $\Phi$ (see the definition of $\Phi$ in $[\mathrm{L}]$ ). By examining Figure 1 we can see that the following diagram commutes:

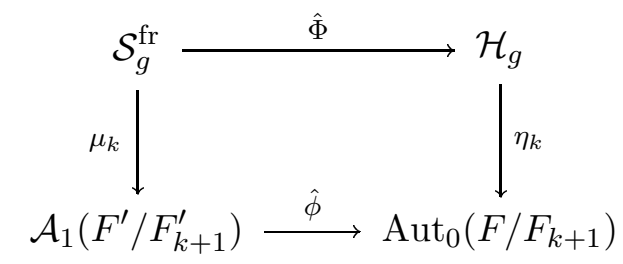

where $\hat{\phi}$ is the injection defined by

$$
\begin{aligned}
& \hat{\phi}\left(\left(\lambda_{i}\right)\right) \cdot y_{i} \equiv \lambda_{i}^{-1} y_{i} \lambda_{i} \quad \bmod F_{k} \\
& \hat{\phi}\left(\left(\lambda_{i}\right)\right) \cdot x_{i} \equiv x_{i} \lambda_{i} \quad \bmod F_{k}
\end{aligned}
$$


As a consequence we see that $\hat{\Phi}$ preserves the weight filtrations and the induced map

$$
\mathcal{S}_{g}^{\mathrm{fr}}[k] / \mathcal{S}_{g}^{\mathrm{fr}}[k+1] \longrightarrow \mathcal{F}_{k}^{w}\left(\mathcal{H}_{g}\right) / \mathcal{F}_{k+1}^{w}\left(\mathcal{H}_{g}\right)=\mathcal{G}_{k}^{w}\left(\mathcal{H}_{g}\right)
$$

corresponds to the monomorphism $\mathrm{D}_{k}\left(H^{\prime}\right) \rightarrow \mathrm{D}_{k}(H)$ induced by inclusion $H^{\prime} \subseteq$ $H$.

Theorem $4 \hat{\Phi}$ is injective.

Proof Let $\overline{\mathcal{H}}_{g}$ denote the subset (it is not a subgroup) of $\mathcal{H}_{g}$ consisting of all $M$ such that $p \eta_{1}(M) i$ is an isomorphism, where $i: H^{\prime} \rightarrow H$ is the inclusion and $p: H \rightarrow H^{\prime}$ is the projection with $p\left(x_{i}\right)=1, p\left(y_{i}\right)=y_{i}$. Note that $\hat{\Phi}\left(\mathcal{S}_{g}^{\mathrm{fr}}\right) \subseteq \overline{\mathcal{H}}_{g}$. We will define a "retraction" $\rho: \overline{\mathcal{H}}_{g} \rightarrow \mathcal{S}_{g}^{\mathrm{fr}}$, which is not a homomorphism, but will satisfy $\rho \circ \hat{\Phi}=$ id and so prove the theorem.

We identify $I \times D_{g}$ with the complement of the trivial framed string link. Now consider an imbedding of $D_{g}$ into $\frac{1}{2} \times D_{g}$ defined by removing a thin collar of the entire boundary of $\frac{1}{2} \times D_{g}$. Thus we obtain an imbedding of $D_{g}$ into the interior of $I \times D_{g}$. Now let $H_{g}$ denote the solid handlebody of genus $g$ whose boundary is $\Sigma_{g} \supseteq \Sigma_{g, 1}$. We will make this identification so that the $y_{i}$ represent a basis for $\pi_{1}\left(H_{g}\right)$, which we identify with $F^{\prime}$, and the $x_{i}$ correspond to the meridians of the handles of $H_{g}$. We can imbed $H_{g}$ into $I \times D_{g}$ as a thickening of the imbedded $D_{g}$ and so that $D_{g} \subseteq \Sigma_{g, 1} \subseteq H_{g}$ is the imbedding which we used to define $\hat{\Phi}$.

Now suppose $M$ represents an element of $\mathcal{H}_{g}$. Then we can cut open $I \times D_{g}$ along the imbedded $\Sigma_{g, 1}$ and insert a copy of $M$ so that $\Sigma^{-} \subseteq H_{g}$. If we identify $I \times D_{g}$ with the complement of the trivial framed string link then our newly constructed manifold is identified with the complement of some string link which lies in a homology 3 -ball precisely when $M \in \overline{\mathcal{H}}_{g}$. We take this to represent $\rho(M)$. It is not hard to see that $\rho \circ \hat{\Phi}=\mathrm{id}$.

Remark 3.1 Note that $\overline{\mathcal{H}}_{g}=\eta_{1}^{-1}(A)$, where $A$ is the subset of $\operatorname{Sp}(H)$ consisting of all $h$ such that $p \circ h \circ i$ is an isomorphism. It is not hard to see that $A \cong P \times Q$, where $P, Q$ are subgroups of $\operatorname{Sp}(H)$ defined as follows: $h \in P$ if and only if $h(L)=L$, where $L=\operatorname{Ker} p$, and $h \in Q$ if and only if $h \mid H^{\prime}=$ id. The bijection is defined by multiplication $\left(h_{1}, h_{2}\right) \mapsto h_{1} \cdot h_{2}$. This product decomposition of $A$ lifts to a product decomposition of $\overline{\mathcal{H}}_{g}$ - see Corollary 3.3.

Remark 3.2 Since $\rho$ is a retraction, $\rho\left(\overline{\mathcal{H}}_{g} \cap \Gamma_{g, 1}\right)$ certainly contains $\mathcal{P}_{g}^{\text {fr }}$, but also contains, for example, the Whitehead string link, Figure 8. 


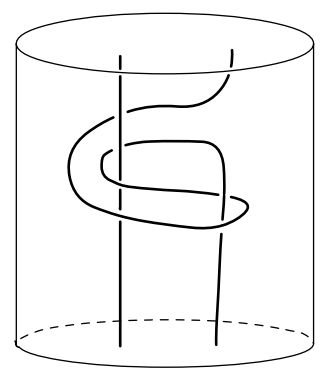

Figure 8

Generally $\sigma \in \rho\left(\overline{\mathcal{H}}_{g} \cap \Gamma_{g, 1}\right)$ if and only if $\sigma$ is represented by a framed string link whose complement is diffeomorphic to $H_{g}$ (i.e. the complement of the trivial string link).

Question 3 What is $\hat{\Phi}^{-1}\left(\Gamma_{g, 1}\right)$ ? Clearly $\mathcal{P}_{g}^{\mathrm{fr}} \subseteq \hat{\Phi}^{-1}\left(\Gamma_{g, 1}\right) \subseteq \rho\left(\overline{\mathcal{H}}_{g} \cap \Gamma_{g, 1}\right)$.

\subsection{Boundary homology cylinders}

Let $M$ be a homology cylinder over $\Sigma_{g, 1}$ and so $\partial M=\Sigma^{+} \cup \Sigma^{-}$. We can also write $\Sigma^{+} \cup \Sigma^{-}=\partial H^{+} \amalg H^{-}$, where $H^{ \pm}$are two copies of $H_{g}$ and $\amalg$ denotes boundary connected sum. Let $V$ be the closed manifold $M \cup\left(H^{+} \amalg\right.$ $\left.H^{-}\right)$. We will say that $M$ is a boundary homology cylinder if $V$ bounds a compact orientable manifold $W$ such that the inclusions $H^{ \pm} \subseteq W$ are homology equivalences. the boundary homology cylinders define a subgroup $\mathcal{H}_{g}^{B} \subseteq \mathcal{H}_{g}$. Note that $\eta_{1}\left(\mathcal{H}_{g}^{B}\right) \subseteq P$ and so $\mathcal{H}_{g}^{B} \subseteq \overline{\mathcal{H}}_{g}$. In fact, since $P \cdot A \subseteq A$, we have $\mathcal{H}_{g}^{B} \cdot \overline{\mathcal{H}}_{g} \subseteq \overline{\mathcal{H}}_{g}$, and so the right coset space $\overline{\mathcal{H}}_{g} / \mathcal{H}_{g}^{B}$ is defined.

Theorem $5 \quad$ (a) $\mathcal{H}_{g}^{B} \cap \Gamma_{g, 1}=\Gamma_{g, 1}^{B}$ the subgroup of $\Gamma_{g, 1}$ consisting of diffeomorphisms which extend to diffeomorphisms of $H_{g}$.

(b) $\mathcal{H}_{g}^{B}=\rho^{-1}(0)$, where 0 denotes the trivial string link.

Proof (a) Suppose $h \in \mathcal{H}_{g}^{B} \cap \Gamma_{g, 1}$. Then $h_{*}\left(x_{i}\right) \in \operatorname{Ker}\left\{\pi_{1}\left(\Sigma_{g, 1}\right) \rightarrow \pi_{1}\left(H_{g}\right)\right\}$ and, therefore, by Dehn's lemma, if $D_{i}$ is the meridian disk of $H_{g}$ corresponding to $x_{i}, h\left(D_{i}\right)$ bounds a disk $D_{i}^{\prime} \subseteq H_{g}$ By standard cut and paste techniques we can assume that the $D_{i}^{\prime}$ are disjoint. We can now extend $h$ over each $D_{i}$ by mapping it onto $D_{i}^{\prime}$. Since the complement of $\cup_{i} D_{i}$ is a 3 -ball, as is the complement of $\cup_{i} D_{i}^{\prime}$, we can extend over $H_{g}$. 
(b) Suppose $M \in \mathcal{H}_{g}^{B}$. Let $V$ be a homology bordism from $H_{g}$ to itself, with $\partial V=H^{+} \cup M \cup H^{-}$, where $H^{ \pm}$are two copies of $H_{g}$. Let $W=I \times\left(I \times D_{g}\right) \cup V$, where $V$ is attached to $\left(1 \times H_{g}\right) \subseteq\left(1 \times\left(I \times D_{g}\right)\right)$ along $H^{+}$. Then $W$ is a homology bordism between $0 \times\left(I \times D_{g}\right)$, the complement of the trivial string link (triv in Figure 9), and $C=$ complement of $S$, where $S$ is the string link constructed as the representative of $\rho(M)$. Thus we can fill in $W$ with product strings to yield a concordance from the trivial string link to $S$. See Figure 9

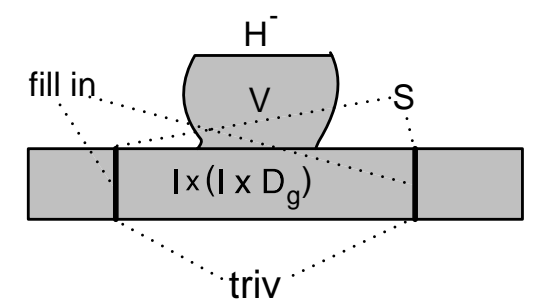

Figure 9: $\rho\left(\mathcal{H}_{g}^{B}\right)=0$

Conversely suppose $M \in \overline{\mathcal{H}}_{g}$ and $\rho(M)=0$. Let $W$ be the complement of a concordance between the trivial string link and $S$, the constructed representative of $\rho(M)$. If $C$ is the complement of $S$, then we can decompose $C=I \times \Sigma_{g, 1} \cup M \cup H_{g}$. Thus

$$
\partial W=H_{g} \cup C=H^{+} \cup M \cup H^{-}
$$

In this way we can see that $W$ is a homology bordism from $H_{g}$ to itself which exhibits $M$ as an element of $\mathcal{H}_{g}^{B}$.

Corollary 3.3 The monomorphisms $\mathcal{H}_{g}^{B} \subseteq \overline{\mathcal{H}}_{g}$ and $\hat{\Phi}: \mathcal{S}_{g}^{\text {fr }} \subseteq \overline{\mathcal{H}}_{g}$ define a bijection

$$
\chi: \mathcal{H}_{g}^{B} \times \mathcal{S}_{g}^{\text {fr }} \rightarrow \overline{\mathcal{H}}_{g}
$$

In particular $\hat{\Phi}$ induces a bijection $\mathcal{S}_{g}^{\text {fr }} \cong \overline{\mathcal{H}}_{g} / \mathcal{H}_{g}^{B}$.

Proof $\chi$ is defined by $\chi(M, S)=M \cdot \hat{\Phi}(S) \in \overline{\mathcal{H}}_{g}$ (see Remark 3.1). To see that $\chi$ is one-one we only need note that $\mathcal{H}_{g}^{B} \cap \hat{\Phi}\left(\mathcal{S}_{g}^{\mathrm{fr}}\right)=1$, which follows from Theorem 5(b) and that $\rho \circ \hat{\Phi}=1$. To prove that $\chi$ is onto we need:

Lemma 3.4 If $M \in \overline{\mathcal{H}}_{g}, S \in \mathcal{S}_{g}^{\text {fr }}$, then $\rho(M \cdot \hat{\Phi}(S))=\rho(M) \cdot S$. 
Proof The idea is given by the schematic pictures below. The first picture shows the complement of $\rho(M \cdot \hat{\Phi}(S))$. C is the complement of the string link $S$ and the shaded region is a product $I \times \Sigma_{g}$. Note that $M \cdot \hat{\Phi}(S)$ can be constructed by stacking $C$ on $M$ along $D_{g} \subseteq \Sigma^{+}$. The second picture gives an alternative view of the first picture which can then be recognized as the complement of $\rho(M) \cdot S$. Filling in the strings completes the proof.
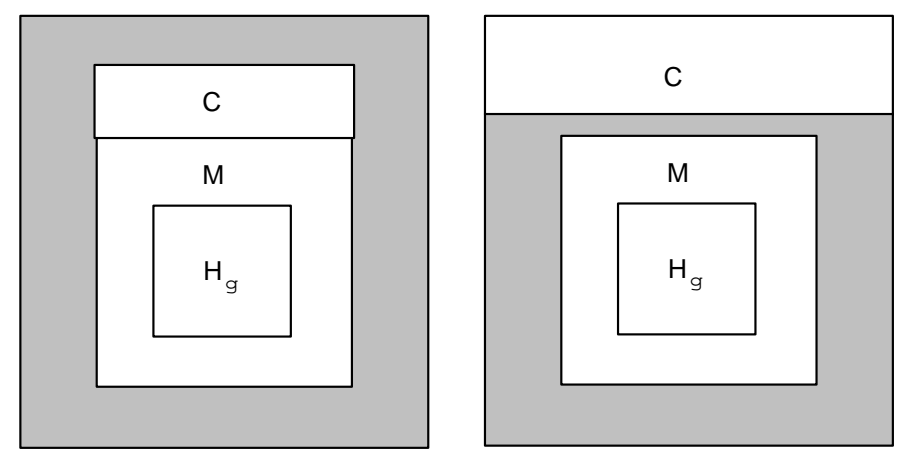

Now suppose $M \in \overline{\mathcal{H}}_{g}$. Then $\rho\left(M \cdot \hat{\Phi}\left((\rho(M))^{-1}\right)=0\right.$, by Lemma 3.4, and $\chi\left(M \cdot \hat{\Phi}\left((\rho(M))^{-1}, \rho(M)\right)=M\right.$.

Remark 3.5 In a recent work of N. Habegger [Ha] a bijection is constructed between the "Torelli" subsemigroup of $\mathcal{C}_{g}$ and the semigroup of $2 g$-strand string links in homology balls with vanishing linking numbers, which although not multiplicative, induces isomorphisms between the associated graded groups of the relative weight filtrations.

The homomorphisms $\eta_{k}: \mathcal{H}_{g} \rightarrow \operatorname{Aut}_{0}\left(F / F_{k+1}\right)$, induce homomorphisms $\eta_{k}^{B}: \mathcal{H}_{g}^{B} \rightarrow \operatorname{Aut}\left(F^{\prime} / F_{k+1}^{\prime}\right)$.

Proposition 3.6 $\eta_{k}^{B}$ is onto, for all $k \geq 1$.

Proof If $h \in \operatorname{Aut}\left(F^{\prime} / F_{k+1}^{\prime}\right)$, then we can lift $h$ to an endomorphism $\bar{h}$ of $F^{\prime}$ and we can realize $\bar{h}$ by an imbedding $H_{g} \subseteq H_{g}$. If $H_{g}^{\prime}$ denotes the imbedded copy of $H_{g}$, then define $V=\left(I \times H_{g}^{\prime}\right) \cup\left(I \times H_{g}\right)$, attaching $1 \times H_{g}^{\prime}$ to $0 \times H_{g}$. $V$ is a homology bordism between $0 \times H_{g}^{\prime}$ and $1 \times H_{g}$ and clearly maps to $h$ under $\eta_{k}^{B}$.

Remark 3.7 The restriction of $\eta_{k}^{B}$ to $\Gamma_{g, 1}^{B}$ is induced by a map $\eta^{B}: \Gamma_{g, 1}^{B} \rightarrow$ Aut $F^{\prime}$ and it is known (see $[\mathrm{G}]$ ) that $\eta^{B}$ is onto. 
To see that $\operatorname{Ker} \eta_{k}^{B}$ is large we consider another imbedding of $\mathcal{S}_{g}^{\text {fr }}$ into $\mathcal{H}_{g}$, $\hat{\Psi}: \mathcal{S}_{g}^{\mathrm{fr}} \rightarrow \mathcal{H}_{g}$, "dual" to $\hat{\Phi}$. This is defined in the same way as $\hat{\Phi}$-from an imbedding $D_{g} \subseteq \Sigma_{g, 1}$ - except that we now choose the imbedding so that the meridians of the holes in $D_{g}$ map onto the meridians of the handles corresponding to $\left\{x_{i}\right\} \subseteq F$. If $F^{\prime \prime}$ is the free group with basis $\left\{x_{i}\right\}$, then we have an injection $\hat{\psi}: \mathcal{A}_{1}\left(F^{\prime \prime} / F_{q}^{\prime \prime}\right) \rightarrow \operatorname{Aut}_{0}\left(F / F_{q}\right)$, defined by

$$
\begin{aligned}
\hat{\psi}\left(\left(\lambda_{i}\right)\right) \cdot x_{i} & \equiv \lambda_{i}^{-1} x_{i} \lambda_{i} \quad \bmod F_{q} \\
\hat{\psi}\left(\left(\lambda_{i}\right)\right) \cdot y_{i} & \equiv \lambda_{i} y_{i} \quad \bmod F_{q}
\end{aligned}
$$

There is a commutative diagram

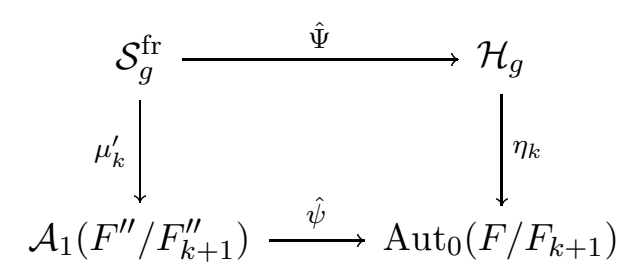

Theorem 6 (a) $\hat{\Psi}\left(\mathcal{S}_{g}^{\text {fr }}\right) \subseteq \mathcal{H}_{g}^{B}$.

(b) $\eta_{k}^{B} \circ \hat{\Psi}=0$ for all $k$.

Proof (a) Write $H_{g}=D^{3} \cup H$, where $H$ represents the $g$ disjoint handles. Then the imbedding $D_{g} \subseteq \Sigma_{g, 1}$ which defines $\hat{\Psi}$ can be regarded as the restriction of an identification of $D^{2}$ with the upper hemisphere $D_{+}^{2}$ of $D^{3}$ - the holes in $D^{2}$ which are removed to obtain $D_{g}$ are the disks along which one end of each handle of $H$ is attached to $D^{3}$. Now consider $I \times D^{2} \subseteq I \times D^{3}$ and a framed string link $S \subseteq I \times D^{2}$. We create a manifold $V$ by attaching $I \times H$ to $I \times D^{3}$ by attaching one end of each handle to $S$ and the other to the trivial framed string link in $I \times D_{-}^{2}$. It is not difficult to see that this is a homology bordism from $H_{g}$ to itself which extends $\hat{\Psi}(S)$.

(b) This follows directly from Equation (3.3) and diagram (3.3), since $\lambda_{i} \in$ $F^{\prime \prime}$.

Question 4 Describe $\bigcap_{k} \operatorname{Ker} \eta_{k}^{B}$. It is known (see [Lu]) that the kernel of $\eta^{B}$ is generated by so-called twist automorphisms, i.e. Dehn twists along properly imbedded 2-disks in $H_{g}$. 


\section{A Lagrangian filtration of the mapping class group}

\subsection{Definition of the filtration}

Let $H_{g}$ and $\Sigma_{g, 1}$ be as above. We define a new filtration of $\Gamma_{g, 1}$. Let $p: F \rightarrow F^{\prime}$ the epimorphism induced by the inclusion $\Sigma_{g, 1} \subseteq H_{g}$, i.e. $p\left(x_{i}\right)=1, p\left(y_{i}\right)=y_{i}$. We define $\mathcal{L}_{g}[k] \subseteq \Gamma_{g, 1}$, for $k \geq 1$, by the condition that $h \in \mathcal{L}_{g}[k]$ if and only if $p \circ h\left(x_{i}\right) \in F_{k+1}^{\prime}$ for all $i$. It is not hard to see that these are subgroups. Set $\mathcal{L}_{g}[\infty]=\bigcap_{k} \mathcal{L}_{g}[k]=\{h \mid \operatorname{Ker} p \subseteq \operatorname{Ker} p \circ h\}$. Note that $h \in \mathcal{L}_{g}[k](k \leq \infty)$ induces an automorphism of $F^{\prime} / F_{k+1}^{\prime}$ and, therefore, of $H^{\prime}=H_{1}\left(F^{\prime}\right)$. This defines a homomorphism $\tau_{k}: \mathcal{L}_{g}[k] \rightarrow \operatorname{Auto}\left(H^{\prime}\right)$. We define $\hat{\mathcal{L}}_{g}[k]=\operatorname{Ker} \tau_{k}$.

We identify some of these groups. Let $L=\operatorname{Ker}\left\{H \rightarrow H^{\prime}\right\}$, a Lagrangian subgroup of the symplectic space $H$. Let $\operatorname{Sp}(H)$ denote the group of symplectic automorphisms of $H$. Let $\tau: \Gamma_{g, 1} \rightarrow \mathrm{Sp}(H)$ denote the standard epimorphism. Let $P(L) \subseteq \operatorname{Sp}(H)$ be the subgroup of all $\sigma$ such that $\sigma(L)=L$ and $P_{0}(L) \subseteq$ $P(L)$ those $\sigma$ such that $\sigma \mid L=\operatorname{id}_{L}$. then $\mathcal{L}_{g}[1]=\tau^{-1}(P(L))$ and $\hat{\mathcal{L}}_{g}[1]=$ $\tau^{-1}\left(P_{0}(L)\right)$, which was denoted $\overline{\mathcal{L}}_{g}^{L}$ in [GL1].

Proposition $4.1 \quad$ (a) $\tau_{k}$ is onto for all $k \leq \infty$.

(b) $\hat{\mathcal{L}}_{g}[2]$ is the subgroup generated by Dehn twists on simple closed curves representing elements of $L$. This is the subgroup denoted $\mathcal{L}_{g}^{L}$ in [GL1].

(c) $\mathcal{L}_{g}[\infty]=\Gamma_{g, 1}^{B}$.

Proof (a) Since $\Gamma_{g, 1}^{B} \subseteq \mathcal{L}_{g}[k]$ for all $k$, this follows immediately from the fact that $\eta^{B}$ is onto $[\mathrm{G}]$.

(b) Note that $h \in \hat{\mathcal{L}}_{g}[2]$ iff. $h\left(x_{i}\right) x_{i}^{-1} \in\left(F_{3} \cdot \Lambda\right) \cap F_{2}$ for all $i$, where $\Lambda=\operatorname{Ker} p$, the normal closure of $\left\{x_{i}\right\}$. To see this first note that $h \in \mathcal{L}_{g}[2]$ iff. $h\left(x_{i}\right) x_{i}^{-1} \in$ $F_{3} \cdot \Lambda$ for every $i$. Secondly note that $h \in \operatorname{Ker} \tau_{k}$ iff. $h\left(y_{i}\right) \equiv y_{i} \bmod F_{2}$ for all $i$-but this is equivalent to $h\left(x_{i}\right) \equiv x_{i} \bmod F_{2}$ for all $i$, since, if a symplectic matrix has the form $\left(\begin{array}{cc}A & B \\ 0 & C\end{array}\right)$, it follows that $A=I$ iff. $C=I$.

Now note that, as a consequence of [L, Cor. 2], $h \in \mathcal{L}_{g}^{L}$ iff. $h\left(x_{i}\right) x_{i}^{-1} \in\left[F, \Lambda \cdot F_{2}\right]$ for all $i$. Thus, since $\left(F_{3} \cdot \Lambda\right) \cap F_{2}=F_{3} \cdot\left(F_{2} \cap \Lambda\right)$ and $\left[F, \Lambda \cdot F_{2}\right]=F_{3} \cdot[F, \Lambda]$, (b) will follow from

Lemma $4.2 \quad F_{2} \cap \Lambda=[F, \Lambda]$. 
Proof By Hopf's theorem the quotient $\frac{F_{2} \cap \Lambda}{[F, \Lambda]}=H_{2}(F / \Lambda)$. But $F / \Lambda \cong F^{\prime}$, which is a free group and so $H_{2}(F / \Lambda)=0$.

(c) If $h \in \mathcal{L}_{g}[\infty]$, then $h_{*}\left(x_{i}\right) \in \Lambda$, for all $i$. We can thus apply the argument in the proof of Theorem 5(a).

Define a function $J_{k}^{L}: \mathcal{L}_{g}[k] \rightarrow \operatorname{Hom}\left(L, \mathrm{~L}_{k+1}\left(H^{\prime}\right)\right) \cong H^{\prime} \otimes \mathrm{L}_{k+1}\left(H^{\prime}\right)$ by $J_{k}^{L}(h)$. $a=p(h(\alpha))$, where $\alpha \in \Lambda$ is any lift of $a \in L$. Note that the symplectic form on $H$ induces an identification of $H^{\prime}$ with the dual space of $L$. Furthermore if $h_{*}: H \rightarrow H$ is the induced automorphism, then $h_{*}(L)=L$ and so there is an induced automorphism of $H^{\prime}$ and of $\mathrm{L}_{k}\left(H^{\prime}\right)$, both of which we also denote by $h_{*}$.

Proposition $4.3 \quad$ (a) $J_{k}^{L}\left(\hat{\mathcal{L}}_{g}[k]\right) \subseteq \mathrm{D}_{k}\left(H^{\prime}\right)$.

(b) $J_{k}^{L}\left(h_{1} \circ h_{2}\right)=\left(h_{2 *} \otimes 1\right) J_{k}^{L}\left(h_{1}\right)+\left(1 \otimes h_{1 *}\right) J_{k}^{L}\left(h_{2}\right)$. Therefore $J_{k}^{L} \mid \hat{\mathcal{L}}_{g}[k]$ is a homomorphism.

(c) $\mathcal{L}_{g}[k+1]=\operatorname{Ker} J_{k}^{L}$.

Proof (a) If $h \in \mathcal{L}_{g}[k]$ then $J_{k}^{L}(h)=\sum_{i} y_{i} \otimes p h_{*}\left(x_{i}\right)$. We will abuse notation and allow ourselves to denote the induced bases of $L$ and $H^{\prime}$ by $\left\{x_{i}\right\}$ and $\left\{y_{i}\right\}$, respectively. If $h \in \hat{\mathcal{L}}_{g}[k]$, then $p h_{*}\left(y_{i}\right)=y_{i}$ in $H^{\prime}$ and so

$$
\begin{aligned}
\gamma_{k}^{\prime} J_{k}^{L}(h) & =\prod_{i}\left[y_{i}, p h_{*}\left(x_{i}\right)\right]=\prod_{i}\left[p h_{*}\left(y_{i}\right), p h_{*}\left(x_{i}\right)\right] \\
& =p h_{*} \prod_{i}\left[y_{i}, x_{i}\right]=p \prod_{i}\left[y_{i}, x_{i}\right]=1
\end{aligned}
$$

in $F_{k+2} / F_{k+3}$, since $h_{*} \prod_{i}\left[y_{i}, x_{i}\right]=\prod_{i}\left[y_{i}, x_{i}\right]$.

(b) Let $\alpha \in \Lambda$ be a lift of $a \in L$. Then we can write $h_{2}(\alpha)=\alpha_{1} \alpha^{\prime}$ for some $\alpha_{1} \in \Lambda$ and $\alpha^{\prime} \in F^{\prime} \subseteq F$, where the latter inclusion is some splitting of $p$. Since $h_{2} \in \mathcal{L}_{g}[k]$ we can choose $\alpha^{\prime} \in F_{k+1}^{\prime}$. If $a_{1}$ is the homology class of $\alpha_{1}$ in $L$, then $a_{1}=h_{2 *}(a)$ and so $p h_{1}\left(\alpha_{1}\right)$ represents $J_{k}^{L}\left(h_{1}\right) \cdot\left(h_{2 *} a\right)$. If $\alpha^{\prime}$ represents $a^{\prime} \in F_{k+1}^{\prime} / F_{k+2}^{\prime}=\mathrm{L}_{k+1}\left(H^{\prime}\right)$, then $a^{\prime}=J_{k}^{L}\left(h_{2}\right) \cdot a$. Thus $p h_{1}\left(\alpha^{\prime}\right)$ represents $h_{1 *}\left(J_{k}^{L}\left(h_{2}\right) \cdot a\right)$.

From these observations we conclude that $J_{k}^{L}\left(h_{1} \circ h_{2}\right) \cdot a$, which is represented by $p\left(h_{1} h_{2}(\alpha)\right)=p h_{1}\left(\alpha_{1}\right) p h_{1}\left(\alpha^{\prime}\right)$ is given by

$$
J_{k}^{L}\left(h_{1} \circ h_{2}\right) \cdot a=J_{k}^{L}\left(h_{1}\right) \cdot h_{2 *} a+h_{1 *} J_{k}^{L}\left(h_{2}\right) \cdot a
$$

(c) This is immediate. 


\subsection{An estimate of $\operatorname{Im} J_{k}^{L}$}

We make use of the imbedding $\Phi: \mathcal{P}_{g}^{\mathrm{fr}} \rightarrow \Gamma_{g, 1}$.

Theorem $7 \quad \Phi$ induces imbeddings

$\Phi_{k}:\left(\mathcal{P}_{g}^{f r}\right)_{k+1} /\left(\mathcal{P}_{g}^{f r}\right)_{k+2} \hookrightarrow \hat{\mathcal{L}}_{g}[k] / \hat{\mathcal{L}}_{g}[k+1] \cong \mathcal{L}_{g}[k] / \mathcal{L}_{g}[k+1] \hookrightarrow \mathrm{D}_{k}\left(H^{\prime}\right) \quad(k \geq 2)$

where $\left(\mathcal{P}_{g}^{\text {fr }}\right)_{q}$ is the $q$-th lower central series subgroup of $\mathcal{P}_{g}^{\text {fr }}$.

The composite imbedding $\left(\mathcal{P}_{g}^{f r}\right)_{k+1} /\left(\mathcal{P}_{g}^{\text {fr }}\right)_{k+2} \hookrightarrow \mathrm{D}_{k}\left(H^{\prime}\right)$ is described as follows. If $\beta \in\left(\mathcal{P}_{g}^{f r}\right)_{k+1}$ is specified by longitude elements $\lambda_{1}, \ldots, \lambda_{g} \in F_{k+1}^{\prime}$, then $\beta$ maps to $\sum_{i} y_{i} \otimes\left[\lambda_{i}\right]$, where $y_{i}$ is a basis of $H^{\prime}$ and $\left[\lambda_{i}\right] \in F_{k+1}^{\prime} / F_{k+2}^{\prime} \stackrel{\cong}{\cong} \mathrm{L}_{k+1}\left(H^{\prime}\right)$ denotes the coset of $\lambda_{i}$.

From this we conclude that $\operatorname{rank} \mathcal{L}_{g}[k] / \mathcal{L}_{g}[k+1] \geq r(g, k)$, where $r(g, k)=$ $\operatorname{rank}\left(\mathcal{P}_{g}^{\mathrm{fr}}\right)_{k+1} /\left(\mathcal{P}_{g}^{\mathrm{fr}}\right)_{k+2}$ is explicitly computable (see the discussion in [L]). The gap between $r(g, k)$ and $\operatorname{Ker} \gamma_{k}: H^{\prime} \otimes \mathrm{L}_{k+1}\left(H^{\prime}\right) \rightarrow \mathrm{L}_{k+2}\left(H^{\prime}\right)$ is also explicitly computable. For example if $k=2$ it is $\frac{1}{6}\left(g^{3}-g\right)$ and for $k=3$ it is $\frac{1}{8}\left(g^{3}-\right.$ $g)(g-2)$-for $k=1$ it is zero.

Proof Recall the definition of $\phi$ from Equation (3.2).

It is well-known that $\beta \in\left(\mathcal{P}_{g}^{\text {fr }}\right)_{q}$, the $q$-th term of the lower central series, if and only if every $\lambda_{i} \in F_{q}^{\prime}$ - see e.g. [F]. We therefore have $\Phi\left(\left(\mathcal{P}_{g}^{\mathrm{fr}}\right)_{k+1}\right) \subseteq \mathcal{L}_{g}[k]$ and the induced $\Phi_{k}$ is injective. That $\hat{\mathcal{L}}_{g}[k] / \hat{\mathcal{L}}_{g}[k+1] \cong \mathcal{L}_{g}[k] / \mathcal{L}_{g}[k+1]$ follows from Proposition 4.1(a).

It also follows directly from the definitions that the composite $\left(\mathcal{P}_{g}^{\mathrm{fr}}\right)_{k+1} \rightarrow$ $\mathcal{L}_{g}[k] \rightarrow H^{\prime} \otimes \mathrm{L}_{k+1}\left(H^{\prime}\right)$ is as claimed.

\section{$5 \quad$ Lagrangian filtration of $\mathcal{H}_{g}$}

We can extend the Lagrangian filtration over $\mathcal{H}_{g}$ in a natural way, using the $\left\{\eta_{k}\right\}$. Set $\mathcal{F}_{k}^{L}\left(\mathcal{H}_{g}\right)=\left\{M \in \mathcal{H}_{g}: p \eta_{k}(M) \mid F^{\prime \prime}=0\right\}$, for $k \geq 1$, where $p: F / F_{k+1} \rightarrow F^{\prime} / F_{k+1}^{\prime}$ is the projection and $F^{\prime \prime} \subseteq F$ via $x_{i} \rightarrow x_{i}$. These are subgroups and obviously $\mathcal{F}_{k}^{w}\left(\mathcal{H}_{g}\right) \subseteq \mathcal{F}_{k}^{L}\left(\mathcal{H}_{g}\right), \mathcal{F}_{k}^{L}\left(\mathcal{H}_{g}\right) \cap \Gamma_{g, 1}=\mathcal{L}_{g}[k]$ and $\mathcal{H}_{g}^{B} \subseteq$ $\mathcal{F}_{k}^{L}\left(\mathcal{H}_{g}\right)$ for every $k$. Now $\eta_{k}$ induces a map $\eta_{k}^{\prime}: \mathcal{F}_{k}^{L}\left(\mathcal{H}_{g}\right) \rightarrow \operatorname{Aut}\left(F^{\prime} / F_{k+1}^{\prime}\right)$ and clearly $\eta_{k}^{\prime} \mid \mathcal{H}_{g}^{B}=\eta_{k}^{B}$. Thus $\eta_{k}^{\prime}$ is onto by Proposition 3.6.

We can also define $\hat{\mathcal{F}}_{k}^{L}\left(\mathcal{H}_{g}\right)=\mathcal{F}_{k}^{L}\left(\mathcal{H}_{g}\right) \cap \operatorname{Ker} \hat{\tau}$, where $\hat{\tau}: \mathcal{H}_{g} \rightarrow \operatorname{Sp}(H)$ is the obvious extension of $\tau$. 
The map $J_{k}^{L}: \mathcal{L}_{g}[k] \rightarrow H^{\prime} \otimes L_{k+1}\left(H^{\prime}\right)$ extends to a map $\hat{J}_{k}^{L}: \mathcal{F}_{k}^{L}\left(\mathcal{H}_{g}\right) \rightarrow$ $H^{\prime} \otimes L_{k+1}\left(H^{\prime}\right)$ and the obvious generalization of Proposition 4.3 holds. In particular $\mathcal{F}_{k+1}^{L}\left(\mathcal{H}_{g}\right)=\operatorname{Ker} \hat{J}_{k}^{L}$.

We now address the homomorphism $\hat{\Phi}: \mathcal{S}_{g}^{\mathrm{fr}} \rightarrow \mathcal{H}_{g}$. Recall the filtration $\left\{\mathcal{S}_{g}^{\mathrm{fr}}[k]\right\}$ from Section 3.1.

Theorem $8 \quad \hat{\Phi}\left(\mathcal{S}_{g}^{\text {fr }}[k]\right) \subseteq \hat{\mathcal{F}}_{k}^{L}\left(\mathcal{H}_{g}\right)$ and induces isomorphisms

$$
\mathcal{S}_{g}^{\text {fr }}[k] / \mathcal{S}_{g}^{\text {fr }}[k+1] \cong \hat{\mathcal{F}}_{k}^{L}\left(\mathcal{H}_{g}\right) / \hat{\mathcal{F}}_{k+1}^{L}\left(\mathcal{H}_{g}\right) \cong \mathcal{F}_{k}^{L}\left(\mathcal{H}_{g}\right) / \mathcal{F}_{k+1}^{L}\left(\mathcal{H}_{g}\right) \cong \mathrm{D}_{k}\left(H^{\prime}\right)
$$

Proof The first assertion is clear from the definitions. The rest of the theorem follows from the observation that the following diagram is commutative

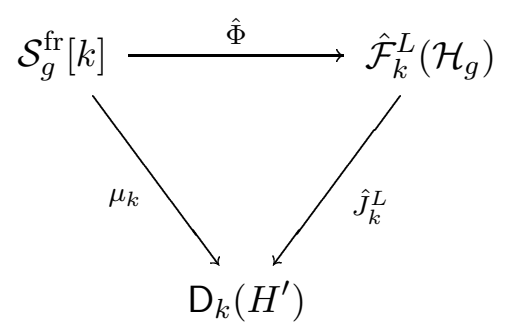

and the fact that $\mu_{k}$ is onto.

We now have $\hat{\Phi}\left(\mathcal{S}_{g}^{\text {fr }}[\infty]\right) \subseteq \hat{\mathcal{F}}_{\infty}^{L}\left(\mathcal{H}_{g}\right)$ and $\mathcal{H}_{g}^{B} \subseteq \mathcal{F}_{\infty}^{L}\left(\mathcal{H}_{g}\right)$. We will see that these two subgroups are independent and generate $\mathcal{F}_{\infty}^{L}\left(\mathcal{H}_{g}\right)$.

Recall the bijection $\chi: \mathcal{H}_{g}^{B} \times \mathcal{S}_{g}^{\mathrm{fr}} \rightarrow \overline{\mathcal{H}}_{g}$ from Corollary 3.3 .

\section{Theorem 9}

$$
\chi\left(\mathcal{H}_{g}^{B} \times \mathcal{S}_{g}^{\text {fr }}[k]\right)=\mathcal{F}_{k}^{L}\left(\mathcal{H}_{g}\right)
$$

for every $2 \leq k \leq \infty$. In particular $\mathcal{F}_{k}^{L}\left(\mathcal{H}_{g}\right)$ is generated by the independent subgroups $\mathcal{H}_{g}^{B}$ and $\hat{\Phi}\left(\mathcal{S}_{g}^{\text {fr }}[k]\right)$.

Proof We need to show that $\chi\left(\mathcal{H}_{g}^{B} \times \mathcal{S}_{g}^{\mathrm{fr}}[k]\right)=\mathcal{F}_{k}^{L}\left(\mathcal{H}_{g}\right)$. First note that $\chi\left(\mathcal{H}_{g}^{B} \times \mathcal{S}_{g}^{\mathrm{fr}}[k]\right) \subseteq \mathcal{F}_{k}^{L}\left(\mathcal{H}_{g}\right)$-this follows from Theorem 8. For the ontoness we can apply the ontoness argument of Corollary 3.3 with the extra fact that 
$\rho\left(\mathcal{F}_{k}^{L}\left(\mathcal{H}_{g}\right)\right) \subseteq \mathcal{S}_{g}^{\mathrm{fr}}[k]$. This follows from the observation that the following diagram is commutative

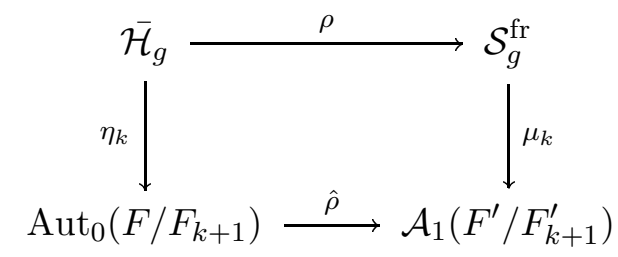

where $\hat{\rho}$ is defined by $\hat{\rho}(h)=\left(p h\left(x_{i}\right)\right)$.

Note that $\hat{\Phi}\left(\mathcal{S}_{g}^{\mathrm{fr}}[\infty]\right) \subseteq \operatorname{Ker} \eta_{k}^{\prime}$ and $\eta_{k}^{\prime} \mid \mathcal{H}_{g}^{B}$ is onto, for every $k$.

\section{References}

[BC] J. Birman, R. Craggs, The $\mu$-invariant of 3-manifolds and certain structural properties of the group of homeomorphisms of a closed oriented 2-manifold, Transactions of the American Mathematical Society 237 (1978) 283-309.

$[\mathrm{CM}]$ T. Cochran, P. Melvin, Finite type invariants for 3-manifolds, Inventiones Math.140 (1), (2000), 45-100. .

[F] M. Falk, R. Randell, The lower central series of a fiber-type arrangement, Inventiones Math. 82 (1) (1985), 77-88.

[GGP] S. Garoufalidis, M. Goussarov, M. Polyak, Calculus of clovers and finite type invariants of 3-manifolds, Geometry and Topology 5 (2001) 75-108.

[GL] S. Garoufalidis, J. Levine, Tree-level invariants of three-manifolds, preprint (1999), arxiv:math.GT/9904106.

[GL1] - Finite type 3-manifold invariants, the mapping class group and blinks, J. Diff. Geom. 47 (1997) 257-320.

[G] H. B. Griffiths, Automorphisms of a 3-dimensional handlebody, Abh. Math. Sem. Univ. Hamburg 26(1963) 191-210.

[Go] M. Goussarov, Finite type invariants and $n$-equivalence of 3-manifolds, C. R. Acad. Sci. Paris Ser. I. Math. 329 (1999) 517-522.

[Ha] N. Habegger, Milnor, Johnson and tree-level perturbative invariants, preprint (2000)

www.math.sciences. univ-nantes.fr/ habegger.

[HL] X X. S. Lin, The classification of Links up to Homotopy, Journal of the A.M.S., 4 (2) (1990) 389-419.

[H] K. Habiro, Claspers and finite type invariants of links, Geometry and Topology 4 (2000) 1-83. 
[Hn] R. Hain, Infinitesimal presentations of the Torelli groups, Journal of the AMS, 10 (3) (1997) 597-651.

[J] D. Johnson, A survey of the Torelli group, Contemporary Math. 20 (1983) 163-179.

[L] J. Levine, Pure braids, a new subgroup of the mapping class group and finitetype invariants, Tel Aviv Topology Conference: Rothenberg Festschrift, ed. M. Farber, W. Luck, S. Weinberger, Contemporary Mathematics 231 (1999).

[Lu] E. Luft, Actions of the homeotopy group of an orientable 3-dimensional handlebody, Math. Annalen 234 (1978) 279-292.

[Ma] S. V. Matveev, Generalized surgery of three-dimensional manifolds and representations of homology spheres, Math. Notices Acad. Sci. USSR, 42:2 (1987) 651-656.

[Mo] S. Morita, Abelian subgroups of the mapping class group of surfaces, Duke Mathematical Jl. 70:3 (1993), 699-726.

[M] Structure of the mapping class group of surfaces: a survey and a prospect, Proceedings of the Kirbyfest, ed. J. Haas and M. Scharlemann, Geometry and Topology monographs, vol. 2 (1999) 349-406.

[N] J. Nielsen, Untersuchungen zur Topologie der Geschlössenen Zweiseitigen Flächen I, Acta Mathematica 50 (1927), 189-358.

[O] T. Oda, A lower bound for the graded modules associated with the relative weight filtration on the Teichmuller group, preprint.

[Oh] T. Ohtsuki, Finite type invariants of integral homology 3-spheres, J. Knot Theory and its Rami. 5 (1996), 101-115.

[St] J. Stallings, Homology and central series of groups, Journal of Algebra 2 (1965), 170-181.

Department of Mathematics, Brandeis University

Waltham, MA 02454-9110, USA

Email: levine@brandeis.edu

URL: www.math.brandeis.edu/Faculty/jlevine/

Received: 14 November 2000

Algebraic $\&$ Geometric $\mathcal{T}$ opology, Volume 1 (2001) 